\title{
Output feedback stabilization for uncertain nonlinear time-delay systems subject to input constraints
}

\author{
D.P. GOODALL*† and R. POSTOYAN ${ }^{\ddagger}$ \\ † Control Theory and Applications Centre, Coventry University, \\ Priory Street, Coventry, CV1 5FB, UK \\ ‡ Univ Paris-Sud, LSS, SUPELEC-C.N.R.S, 3 Rue Joliot Curie, \\ 91192 Gif-sur-Yvette Cedex, France
}

2 June 2009

\begin{abstract}
Robust stabilization of a class of imperfectly known systems with time-varying time-delays via output feedback is investigated. The systems addressed are composed of a nonlinear nominal system influenced by nonlinear perturbations which may be time-, state, delayed state, and/or inputdependent. The output of the system is modelled by a nonlinear function, which may depend on the delayed states, and inputs, together with a feed-through term. Using bounding information on the perturbations, in terms of specified growth conditions, classes of unconstrained and constrained output feedback controllers are designed in order to guarantee a prescribed stability property for the closed-loop systems, provided appropriate stability criteria hold. Two stability criterion are given: one in terms of a Linear Matrix Inequality (LMI), the other is algebraic in nature, obtained using a Geršgorin theorem.
\end{abstract}

Keywords: input/control constraints; output feedback stabilization; time-delay systems of the retarded type; uncertain nonlinear systems.

\section{Introduction}

Time-delay systems have received much attention over the last decades (see, for instance, the recent survey papers by Gu et al. [10] and Richard [21]). This interest is motivated by the fact that many physical systems can be modelled by dynamical structures that do not only depend on the states at the present time but also on the past states, such as the internal combustion engine, chemical engineering processes, and population dynamics. When time-delays are considered to be time-varying, some researchers have investigated some stabilization issues (see Fridman et al. [6], He et al. [13], Kao et al. [15], Lam et al. [16], ). Moreover, the dynamics of many complex systems do not only depend on one single time-delay, but also on several time-delays. Thus, a number of authors have focused on the stability of systems with multiple time-delays; for example, see Fridman et al. [6]. In addition, robust control time-delay problems have been studied for perturbed known nominal models, but relatively few studies have considered the case of a nonlinear nominal model (see Clarkson et al. [4], Goodall [8, 9], Kao et al. [15], for example, and also Zhang et al. [28], in which the nonlinear systems have a feedforward structure).

Due to the possible non-availability of the full states of a system, a number of authors have focused on output stabilization problems for uncertain (non)linear systems with time-delays. Output feedback controllers avoid the use of observation techniques, which will increase the order of the system and, hence, the complexity of the problem. Dynamic output stabilizers have been developed, for instance, in Chen et al. [2], de Oliveira et al. [5], Park [19] for linear systems, whilst in Mirkin et al. [18] a class

${ }^{*}$ Corresponding author. Email: d.goodall@coventry.ac.uk 
of adaptive stabilizers is developed. Some researchers have worked on static output controllers for such systems. In Zhang et al. [27] the problem is addressed for a class of nonlinear systems with a delayed input. Based on input-output feedback linearisation, a class of static output controllers is designed in $\mathrm{Wu}$ et al. [26], introducing the notion of constant relative degree. In both works, Zhang et al. [27] and $\mathrm{Wu}$ et al. [26], the output function is delay-free, but that is not always true in practice where the measurement may be delayed; see for instance Germani et al. [7]. In Clarkson et al. [3] the delayed measurement problem is addressed via a class of static output stabilizers, without invoking the concept of constant relative degree.

In this study, the output stabilization problem for a class of nonlinear uncertain time-delay systems is addressed, in which the time-delays, that affect the system dynamics and the output function, are time-varying. Assuming some stability properties on the nominal system hold, classes of static (un)constrained output controllers with memory are designed. The proposed stabilizers can be seen as the adaptation of the state-feedback controllers developed in Clarkson et al. [4]. It is shown that stabilization of the closed-loop system is ensured provided some stability criteria hold that are expressed in terms of either a LMI or an algebraic relation. The stability criteria are delay-dependent since they involve the bound on the time-derivatives of the delays. Here, the work in Clarkson et al. [3] is extended to a larger class of systems affected by a finite number a time-varying delays, and the output function is also time-delay dependent and input dependent; moreover, the sufficient conditions for stabilization are relaxed. An illustrative example is presented that confirms theoretical results for the constrained output stabilization. Note that, at this present time, the authors are not aware of any study on designing a class of constrained static output stabilizers, with feed-through terms, for nonlinear uncertain time-delay systems, which ensures a global asymptotic stability property under appropriate sufficient conditions.

The paper is organised as follows. After having defined the nomenclature and some mathematical preliminaries in Section 2, the problem is clearly stated in Section 3. In Section 4, a class of unconstrained output stabilizers is designed and the stability of the closed-loop system is analysed. The case of constrained feedbacks is treated in Section 5 and the illustrative example is given in Section 6 .

\section{Mathematical preliminaries}

The notation $\mathcal{I}_{n}$ is used to denote the set of integers $\{0, \ldots, n\}$ and $\mathcal{J}_{n}$ represents $\mathcal{I}_{n} \backslash\{0\}$. Let $n, m \in \mathbb{N}$ (the set of natural numbers $\{1,2, \ldots\}$ ), with $n \geq m, \mathbb{R}:=(-\infty, \infty), \mathbb{R}^{+}:=(0, \infty), \mathbb{R}_{0}^{+}:=[0, \infty)$ and suppose the Euclidean inner product (on $\mathbb{R}^{n}$ or $\mathbb{R}^{m}$ as appropriate) and the induced norm are denoted by $\langle\cdot, \cdot\rangle$ and $\|\cdot\|$, respectively. Let $C\left(\mathbb{R}^{n} ; \mathbb{R}^{m}\right)$ denote the space of all continuous functions mapping $\mathbb{R}^{n} \rightarrow \mathbb{R}^{m}$ and $C^{1}\left(\mathbb{R}^{n} ; \mathbb{R}^{m}\right)$ the space of continuous functions with continuous first order partial derivatives. Also, for $p \in \mathbb{N}$ and $a, b \in \mathbb{R}$ with $b>a$, let $L^{p}(a, b)$ denote the space of all functions $t \mapsto x(t) \in \mathbb{R}$ which are defined almost everywhere and measurable in $[a, b)$ and for which $\int_{a}^{b}|x(t)|^{p} \mathrm{~d} t$ exists in the sense of Lebesgue.

If $h: \mathcal{Y}_{1} \rightarrow \mathcal{Y}_{2}$, where $\mathcal{Y}_{1}$ and $\mathcal{Y}_{2}$ are metric spaces, is continuous and if, for any bounded set $\mathcal{B} \subset \mathcal{Y}_{1}$, the closure of $h(\mathcal{B}):=\{h(b): b \in \mathcal{B}\}$ is compact, then $h$ is said to be completely continuous. For a vector field $f \in \mathbb{R}^{n}$ and a function $g \in C^{1}\left(\mathbb{R}^{n} ; \mathbb{R}\right), L_{f} g$ denotes the Lie derivative of $g$ in the direction of $f$, that is $\langle(\nabla g)(x), f(x)\rangle$, where $x \in \mathbb{R}^{n}$ and $(\nabla g)(x)$ denotes the gradient vector field $(\partial g / \partial x)(x)$. The notation $x_{t}=x_{t}(\theta):=x(t+\theta)$, with $\theta \in[-\tau, 0]$ and $\tau \in \mathbb{R}^{+}$, is introduced, which denotes the restriction of $x(\cdot)$ to the interval $[t-\tau, t]$. Moreover, for $i \in \mathcal{J}_{n}, x_{i t}(-\tau)$ denotes $x_{i}(t-\tau)$. Note that if $x_{t} \in C\left([-\tau, 0] ; \mathbb{R}^{n}\right)$, then $\left\|x_{t}\right\|_{\tau}:=\sup _{\theta \in[-\tau, 0]}\|x(t+\theta)\|$. Let $\mathcal{Q}_{A, \tau}^{n}:=\left\{q \in C\left([-\tau, 0] ; \mathbb{R}^{n}\right)\right.$ : $\|q\|<A, 0<A<\infty\}$ denote a set of bounded functions in $C$. In addition, let $\Omega$ be the set of scalar nondecreasing functionals, $\omega \in C(\mathbb{R} ; \mathbb{R})$, such that $\omega(r)>0$ for $r>0$, and $\omega(0)=0$. For $i \in \mathcal{I}_{n}$ $(n \in \mathbb{N}),\left(t, z(t), z_{t}^{(1)}, \ldots, z_{t}^{(i)}\right) \in \mathbb{R} \times \mathbb{R}^{n} \times \mathcal{Q}_{A, \tau}^{n} \times \ldots \times \mathcal{Q}_{A, \tau}^{n}, a \in C\left(\mathbb{R} \times \mathbb{R}^{n} \times \mathcal{Q}_{A, \tau}^{n} \times \ldots \times \mathcal{Q}_{A, \tau}^{n} ; \mathbb{R}^{n}\right)$ and a $C^{1}$ functional $b: \mathbb{R} \times \mathbb{R}^{n} \times \mathcal{Q}_{A, \tau}^{n} \times \ldots \times \mathcal{Q}_{A, \tau}^{n} \rightarrow \mathbb{R}$, let $\left(D_{a} b\right)\left(t, z_{t}\right)$ denote $\sum_{\gamma \in \mathcal{I}_{i}}\left\langle\nabla_{z_{t}^{(i)}} \bar{b}, \bar{a}\right\rangle$, where $z_{t}^{(0)}:=z(t), \bar{a}:=a\left(t, z_{t}^{(0)}, \ldots, z_{t}^{(i)}\right)$ and $\bar{b}:=b\left(t, z_{t}^{(0)}, \ldots, z_{t}^{(i)}\right)$. Also, for two sets $\mathcal{E}, \mathcal{F} \subset \mathbb{R}^{n}$, the notation $\mathcal{E} \subsetneq \mathcal{F}$ is introduced to denote that the set $\mathcal{E}$ is strictly included within the set $\mathcal{F}$, and the notation $\emptyset$ is used to denote an empty set. The notion of distance of a point $a \in \mathbb{R}^{n}$ to a set $\mathcal{S} \subset \mathbb{R}^{n}$ is defined and denoted as $\delta(a, \mathcal{S}):=\inf _{s \in \mathcal{S}} \delta(a, s)$, where $\delta$ denotes some metric on $\mathbb{R}$. The notation $\operatorname{diag}\left(a_{i}\right)\left(i \in \mathcal{I}_{n}\right)$ denotes a square diagonal matrix in which the element in the $i$ th row and $i$ th column is $a_{i} \in \mathbb{R}$. If $P \in \mathbb{R}^{p \times p}, P>0$ denotes that $P$ is positive definite and, for $Q \in \mathbb{R}^{p \times p}$, the statement $P<Q$ denotes that $Q-P$ is positive definite. Let $\sigma_{\min (\max )}(\cdot)$ denote the minimum (maximum) eigenvalue 
of a symmetric positive definite matrix and let $\|A\|:=\sqrt{\sigma_{\max }\left(A^{\mathrm{T}} A\right)}$, where the superscript ' $\mathrm{T}$ ' denotes matrix transposition.

In the ensuing stability analysis, a Geršgorin Theorem will be invoked.

Theorem 1 (Geršgorin Theorem, Varga [24]) Let $A$ be a real square matrix, $A=\left[a_{i, j}\right]$, for all $i, j \in \mathcal{J}_{n}$, with $n \in \mathbb{N}, n>1$ and $a_{i, j} \in \mathbb{R}$. For all $i \in \mathcal{J}_{n}$, the notation $D_{i}$ denotes the circle centered in $a_{i, i}$ with the radius $\sum_{j \in \mathcal{J}_{n}, j \neq i}\left|a_{i, j}\right|$ and $D_{i}$ is called a Geršgorin circle of the matrix A. Then, every eigenvalue of A lies at least in one Geršgorin circle.

\section{Problem statement}

The class of systems considered is composed of a nonlinear functional differential equation of the retarded type with time-varying delays affected by nonlinear perturbations. The output of the systems is modelled by a nonlinear function, dependent on the states and/or the delayed-states, and a feed-through term. The system model is assumed to have the following structure:

$$
\begin{gathered}
\dot{x}(t)=f\left(t, x(t), x_{t}\left(-\tau_{1}(t)\right), \ldots, x_{t}\left(-\tau_{i}(t)\right)\right)+q\left(t, x(t), x_{t}\left(-\tau_{1}(t)\right), \ldots, x_{t}\left(-\tau_{i}(t)\right), u(t)\right) \\
+G(x(t))\left[u(t)+p\left(t, x(t), x_{t}\left(-\tau_{1}(t)\right), \ldots, x_{t}\left(-\tau_{i}(t)\right), u(t)\right)\right], \\
y(t)=h\left(x(t), x_{t}\left(-\tau_{1}(t)\right), \ldots, x_{t}\left(-\tau_{i}(t)\right)\right)+\sigma(u(t)), \quad t>t_{0} \geq 0, \\
x_{t_{0}}(\theta)=\phi(\theta), \theta \in[-\bar{\tau}, 0] \text { with } \phi(0)=x^{0},
\end{gathered}
$$

where $x(t) \in \mathbb{R}^{n}$ is the instantaneous state vector, $u(t) \in \mathbb{R}^{m}$ is the control or input vector, $y(t) \in \mathbb{R}^{p}$ is the output vector, $\tau_{\gamma}(\cdot), \gamma \in \mathcal{J}_{i}$, are delays bounded by $\bar{\tau} \in \mathbb{R}^{+}$and $n, m, p, i \in \mathbb{N}$, with $m \leq n$. With $\left(t_{0}, x^{0}\right) \in \mathbb{R} \times \mathbb{R}^{n}$ specified and $\bar{\tau}>0, \phi \in C\left([-\bar{\tau}, 0] ; \mathbb{R}^{n}\right)$ satisfies the initial condition $x\left(t_{0}\right)=\phi(0)=x^{0}$. In addition, $f: \mathbb{R} \times \mathbb{R}^{n} \times \mathcal{Q}_{A, \bar{\tau}}^{n} \times \ldots \times \mathcal{Q}_{A, \bar{\tau}}^{n} \rightarrow \mathbb{R}^{n}$, which is completely continuous and satisfies $f(t, 0, \ldots, 0) \equiv 0$ for all $t \in\left[t_{0}, \infty\right), G(x)=\left[g_{1}(x) \ldots g_{m}(x)\right]$ which is a matrix-valued function with $g_{k} \in$ $C\left(\mathbb{R}^{n} ; \mathbb{R}^{n}\right), k \in \mathcal{J}_{m}$, and $h \in C\left(\mathbb{R}^{n} \times \mathcal{Q}_{A, \bar{\tau}}^{n} \times \ldots \times \mathcal{Q}_{A, \bar{\tau}}^{n} ; \mathbb{R}^{p}\right)$ which satisfies $h(0, \ldots, 0) \equiv 0$, are assumed to be known. The function $\sigma \in C\left(\mathbb{R}^{m} ; \mathbb{R}^{p}\right)$ represents the feed-through term. The 'uncertainty' functions $p=\left[p_{1}, \ldots, p_{m}\right]^{\mathrm{T}}: \mathbb{R} \times \mathbb{R}^{n} \times \mathcal{Q}_{A, \bar{\tau}}^{n} \times \ldots \mathcal{Q}_{A, \bar{\tau}}^{n} \times \mathbb{R}^{m} \rightarrow \mathbb{R}^{m}$ and $q: \mathbb{R} \times \mathbb{R}^{n} \times \mathcal{Q}_{A, \bar{\tau}}^{n} \times \ldots \times \mathcal{Q}_{A, \bar{\tau}}^{n} \times \mathbb{R}^{m} \rightarrow \mathbb{R}^{n}$ are assumed to be completely continuous and belong to a known non-empty class, which comprise all possible system uncertainty, as well as any known time-dependent and/or nonlinear elements. The form of system (1a-1c) allows one to consider different delays in the dynamic equation (1a) and the output function (1b) which is very useful in practice, since one can consider specific delays in the output during measurements, for instance.

For presentational convenience, let $\tau_{0}, \bar{\tau}_{0}, \hat{\tau}_{0}:=0$. The time-delays $\tau_{\gamma}(\cdot), \gamma \in \mathcal{J}_{i}$, are modelled by continuously differentiable functions satisfying the following conditions:

H1: For all $(t, \gamma) \in\left[t_{0}, \infty\right) \times \mathcal{J}_{i}$, there exist $\bar{\tau}_{\gamma} \geq 0$ and known $\hat{\tau}_{\gamma} \geq 0$ such that,

a) $\tau_{\gamma}(t) \leq \bar{\tau}_{\gamma}$;

b) $\dot{\tau}_{\gamma}(t) \leq \hat{\tau}_{\gamma}<1$.

Thus, in (1c), $\bar{\tau}=\max _{\gamma \in \mathcal{I}_{i}} \bar{\tau}_{\gamma}$.

Remark 1 The conditions $\mathrm{H} 1$ are often specified when investigating systems with time-varying delays using the Lyapunov-Krasovskii functional technique (for example, see Haurani et al. [12]). Alternative conditions, in which the bounding condition on $\dot{\tau}_{\gamma}(t)$ are relaxed, are given by Fridman et al. in [6]. Note that the time-varying delays do not need to be known explicitly, only bounding information on the time-derivative of the delays, namely $\hat{\tau}_{\gamma}$, is supposed to be known.

Consider the nominal time-delay drift system derived from (1a-c), namely the known system with no input, no output and no uncertainty:

$$
\begin{aligned}
& \dot{x}(t)=f\left(t, x(t), x_{t}\left(-\tau_{1}(t)\right), \ldots, x_{t}\left(-\tau_{i}(t)\right)\right), \\
& x_{t_{0}}(\theta)=\phi(\theta), \quad \theta \in[-\bar{\tau}, 0] \text { with } \phi(0)=x^{0} .
\end{aligned}
$$

Since $f$ satisfies, $\forall t \in\left[t_{0}, \infty\right), f(t, 0, \ldots, 0) \equiv 0$, then $x=0$ is a state equilibrium for system (2a-b).

Hypotheses concerning nominal system stability are given $\mathrm{H} 2$ in terms of a known functional. 
H2: Suppose there exist real constants $a_{k}, b_{k} \geq 0, k \in \mathcal{J}_{i}, b_{\left(\gamma, \gamma^{\prime}\right)},\left(\gamma, \gamma^{\prime}\right) \in \mathcal{J}_{i} \times \mathcal{J}_{i}$, and $c>0$. Also, suppose there exist real functions $\omega_{k} \in \Omega$ (with $\Omega$ defined in $\left.\S 2\right), k \in \mathcal{J}_{i+4}, \forall\left(l, l^{\prime}\right) \in \mathcal{I}_{i} \times \mathcal{I}_{i}, d_{\left(l, l^{\prime}\right)}$ : $\mathbb{R} \rightarrow\left[0, \bar{d}_{\left(l, l^{\prime}\right)}\right], 0<\bar{d}_{\left(l, l^{\prime}\right)}<\infty$, with $d_{\left(l, l^{\prime}\right)}=d_{\left(l^{\prime}, l\right)}, s \in C\left(\mathbb{R}^{n} ; \mathbb{R}_{0}^{+}\right)$and a functional $v_{1} \in C^{1}\left(\mathbb{R} \times \mathbb{R}^{n} \times\right.$ $\left.\mathcal{Q}_{A, \bar{\tau}}^{n} \times \ldots \times \mathcal{Q}_{A, \bar{\tau}}^{n} ; \mathbb{R}_{0}^{+}\right)$, such that, for any $0<A<\infty$ and for all $\left(t, \psi(0), \psi\left(-\tau_{1}(t)\right), \ldots \psi\left(-\tau_{i}(t)\right)\right) \in$ $\left[t_{0}, \infty\right) \times \mathbb{R}^{n} \times \mathcal{Q}_{A, \bar{\tau}}^{n} \times \ldots \times \mathcal{Q}_{A, \bar{\tau}}^{n}$,

a) $v_{1}(t, 0, \ldots, 0)=0$

b) $\omega_{1}(\|\psi(0)\|) \leq v_{1}\left(t, \psi(0), \psi\left(-\tau_{1}(t)\right), \ldots \psi\left(-\tau_{i}(t)\right)\right) \leq \omega_{2}\left(\|\psi\|_{\bar{\tau}}\right)$ with $\omega_{1}(r) \rightarrow \infty$ as $r \rightarrow \infty$;

c) along solutions to (2a) and for all $t \in\left[t_{0}, \infty\right)$,

$$
\begin{aligned}
& \frac{\partial v_{1}}{\partial t}\left(t, x(t), x_{t}\left(-\tau_{1}(t), \ldots, x_{t}\left(-\tau_{i}(t)\right)\right)+\left(D_{v_{1}} f\right)\left(t, x_{t}\right) \leq-s^{2}(x(t))\right. \\
& +\sum_{\left(\gamma, \gamma^{\prime}\right) \in \mathcal{J}_{i} \times \mathcal{J}_{n}} b_{\left(\gamma, \gamma^{\prime}\right)} \omega_{\gamma+4}\left(\left|x_{\gamma^{\prime} t}\left(-\tau_{\gamma}(t)\right)\right|\right) \\
& +\sum_{\gamma \in \mathcal{J}_{i}} d_{(0, \gamma)}(t) s(x(t)) s\left(x_{t}\left(-\tau_{\gamma}(t)\right)\right)+\frac{1}{2} \sum_{\left(l, l^{\prime}\right) \in \mathcal{J}_{i} \times \mathcal{J}_{i}} d_{\left(l, l^{\prime}\right)}(t)\left\{\omega_{3}\left(\left\|x_{t}\left(-\tau_{l}(t)\right)\right\|\right) \omega_{3}\left(\left\|x_{t}\left(-\tau_{l^{\prime}}(t)\right)\right\|\right)\right\}^{\frac{1}{2}},
\end{aligned}
$$

almost everywhere, where $\sum_{\gamma \in \mathcal{J}_{i}}\left(1-\hat{\tau}_{\gamma}\right)^{-\frac{1}{2}} \bar{d}_{(0, \gamma)}<1$ and

$$
\begin{aligned}
\left(1-\sum_{\gamma \in \mathcal{J}_{i}}\left(1-\hat{\tau}_{\gamma}\right)^{-\frac{1}{2}} \bar{d}_{(0, \gamma)}\right) s^{2}(x(t)) \geq & c \omega_{3}(\|x(t)\|) \\
& +\sum_{\left(\gamma, \gamma^{\prime}\right) \in \mathcal{J}_{i} \times \mathcal{J}_{n}}\left(1-\hat{\tau}_{\gamma}\right)^{-1} b_{\left(\gamma, \gamma^{\prime}\right)} \omega_{\gamma+4}\left(\left|x_{\gamma^{\prime} t}\left(-\tau_{\gamma}(t)\right)\right|\right) ;
\end{aligned}
$$

d) for all $r \in \mathbb{R}^{n}, s^{2}(r) \leq \omega_{4}(\|r\|)$;

e) $\left\|\nabla_{\psi} v_{1}\left(t, \psi(0), \psi\left(-\tau_{1}(t)\right), \ldots \psi\left(-\tau_{i}(t)\right)\right)\right\| \leq \sum_{\gamma \in \mathcal{I}_{i}} a_{\gamma}\left\{\omega_{3}\left(\left\|\psi\left(-\tau_{\gamma}(t)\right)\right\|\right)\right\}^{\frac{1}{2}}$.

Remark 2 According to Theorem 2.1, Chapter 5 in Hale [11], if the conditions of H2 a)-d) hold, then, invoking Theorem 1, a straightforward analysis shows that, with reference to (2a) and with initial condition (2b), $\{0\}$ is asymptotically stable when $c>\frac{1}{2} \sum_{\left(l, l^{\prime}\right) \in \mathcal{J}_{i} \times \mathcal{J}_{i}}\left(1-\hat{\tau}_{l}\right)^{-\frac{1}{2}} \bar{d}_{\left(l, l^{\prime}\right)}$. It will be shown later, in Section 4 and 5, that the proposed stability criteria require global uniform asymptotic stability of the origin of the nominal system.

Remark 3 The conditions on the system, stated in $\mathrm{H} 2$, allows one to consider a larger class of systems than the hypotheses required in Clarkson et al. [3, 4].

The matched, $p_{k}, k \in \mathcal{J}_{m}$, and the residual, $q$, uncertainties occurring in system (1a) are assumed to satisfy the growth conditions below.

H3: For all $\left(k, t, z_{0}, z_{1}, \ldots, z_{i}, u\right) \in \mathcal{J}_{m} \times\left[t_{0}, \infty\right) \times \mathbb{R}^{n} \times \ldots \times \mathbb{R}^{n} \times \mathbb{R}^{m}$, there exists known continuous functions $\alpha_{k}:\left[t_{0}, \infty\right) \rightarrow\left[0, \bar{\alpha}_{k}\right], \bar{\alpha}_{k} \in \mathbb{R}^{+}, \kappa_{k}:\left[t_{0}, \infty\right) \rightarrow\left[0, \bar{\kappa}_{k}\right]$, with $0 \leq \bar{\kappa}_{k}<1, \alpha:\left[t_{0}, \infty\right) \rightarrow[0, \hat{\alpha}]$, $\hat{\alpha} \in \mathbb{R}^{+}$, and known real constants $\beta_{\gamma, k}, \beta_{\gamma}, \rho_{k} \geq 0, \gamma \in \mathcal{I}_{i}$, such that

a) $\left|p_{k}\left(t, z_{0}, z_{1}, \ldots, z_{i}, u\right)\right| \leq \alpha_{k}(t)+\sum_{\gamma \in \mathcal{I}_{i}} \beta_{\gamma, k}\left\{\omega_{3}\left(\left\|z_{\gamma}\right\|\right)\right\}^{\frac{1}{2}}+\kappa_{k}(t)\left|u_{k}(t)\right|$

b) $\left\|q\left(t, z_{0}, z_{1}, \ldots, z_{i}, u\right)\right\| \leq \alpha(t)+\sum_{\gamma \in \mathcal{I}_{i}} \beta_{\gamma}\left\{\omega_{3}\left(\left\|z_{\gamma}\right\|\right)\right\}^{\frac{1}{2}}+\sum_{k \in \mathcal{J}_{m}} \rho_{k} \sum_{\gamma \in \mathcal{I}_{i}}\left|\left\langle\nabla_{z_{\gamma}} v_{1}\left(t, z_{0}, z_{1}, \ldots, z_{i}\right), g_{k}\left(z_{0}\right)\right\rangle\right|$.

The aim of this work is to guarantee a global uniform asymptotic stability property for some prescribed sets, that include the state origin, and global attractivity results for the closed-system (1a-b), subject to the given initial conditions (1c), using a class of output controllers.

Firstly, definitions of global uniform asymptotic stability and global attractivity of a compact set are presented (see Definitions 3.2.3, 3.2.5 and 3.2.12, §3.2, Chapter 3, in Michel et al. [17]). Let $\mathcal{S} \subset \mathbb{R}^{n}$ be a compact non-empty set, containing $\{0\}$. 
Definition $1 \mathcal{S}$ is an invariant set with respect to system $(1 \mathrm{a}-\mathrm{b})$ if $\phi(0) \in \mathcal{S}$ implies that $x\left(t, t_{0}, \phi\right) \in \mathcal{S}$ for all $t \geq t_{0}$.

Definition 2 If $\mathcal{S}$ is invariant with respect to (1a-b), then $\mathcal{S}$ is a uniformly asymptotically stable invariant set for system (1a-b) if the following hold

(i) Existence and Continuation of Solutions: for each $\left(t_{0}, \phi, x^{0}\right) \in \mathbb{R}_{0}^{+} \times \mathcal{Q}_{A}^{\bar{\tau}} \times \mathbb{R}^{n}$, there exists a solution $x\left(\cdot, t_{0}, \phi\right)$ defined on $\left[t_{0}-\tau, t_{1}\right)\left(t_{1}>t_{0}-\tau\right)$ and every such solution can be extended into a solution on $\left[t_{0}-\tau, \infty\right)$.

(ii) Uniform Stability of $\mathcal{S}$ : for each $\varepsilon>0$ and for every $t_{0} \in \mathbb{R}_{0}^{+}$, there exists $\Delta=\Delta(\varepsilon)>0$, (which is independent of $\left.t_{0}\right)$, such that whenever $\delta\left(x^{0}, \mathcal{S}\right)<\Delta, \delta\left(x\left(t, t_{0}, \phi\right), \mathcal{S}\right)<\varepsilon$ for all $t \geq t_{0}$ on every solution $x\left(\cdot, t_{0}, \phi\right)$ of $(1 \mathrm{a}-\mathrm{b})$.

(iii) Uniform Attractivity of $\mathcal{S}$ : for each $\varepsilon>0, t_{0} \in \mathbb{R}_{0}^{+}$and $x^{0}$, there exists $\Delta>0$, (which is independent of $t_{0}$ and $\left.\varepsilon\right)$, and a real number $T_{\varepsilon}\left(x^{0}\right) \geq 0$, independent of $t_{0}$, such that if $\delta\left(x^{0}, \mathcal{S}\right)<\Delta$ then $\delta\left(x\left(t, t_{0}, \phi\right), \mathcal{S}\right)<\varepsilon$ for all $t \geq t_{0}+T_{\varepsilon}\left(x^{0}\right)$ on every solution $x\left(\cdot, t_{0}, \phi\right)$ of $(1 \mathrm{a}-\mathrm{b})$.

Moreover, if the above hold with $\Delta$ arbitarily large then $\mathcal{S}$ is said to be a globally uniformly asymptotically stable invariant set for system (1a-b).

Attention is focused on two cases, namely utilising unconstrained and constrained controllers. Sufficient conditions for the closed-loop system are then derived, that ensure desired stability properties hold. The following theorem will be used for the stability analysis in the sequel. This theorem is a consequence of Theorem 4.4.1, §4.4, Chapter 4, in Michel et al. [17] (see, also, Theorem 6.2.22, $\S 6.2$, Chapter 6, in Michel et al. [17]).

Theorem 2 Let $\mathcal{S}$ be a non-empty, compact set satisfying $\{0\} \subset \mathcal{S} \subset \mathbb{R}^{n}$. Consider system (1a-c) and suppose there exist $\pi_{i} \in \Omega$, with $\pi_{i}(r) \rightarrow \infty$ as $r \rightarrow \infty$, for $i=1,2,3$, and a $C^{1}$ functional $w(t, \psi)$ such that, for all $(t, \psi) \in\left[t_{0}, \infty\right) \times \mathcal{Q}_{A, \bar{\tau}}^{n}$,

(i) $\pi_{1}(\delta(\psi(0), \mathcal{S})) \leq w(t, \psi) \leq \pi_{2}\left(\sup _{r \in[-\bar{\tau}, 0]} \delta(\psi(r), \mathcal{S})\right)$;

(ii) along solutions to $(1 \mathrm{a}-\mathrm{b}), \dot{w}\left(t, x_{t}\right)+\pi_{3}(\delta(x(t), \mathcal{S})) \leq 0$,

then the set $\mathcal{S}$ is globally uniformly asymptotically stable.

Moreover, Theorem 2 can be adapted (in view of Theorem 2.14, $\S 2.5$, and Corollary 2.18, $\S 2.6$, Chapter 2 , in $\mathrm{Qu}[20])$ to obtain sufficient conditions for solutions to be globally uniformly bounded and an attractivity result.

Theorem 3 Consider system (1a-c) and suppose there exist

- $\pi_{i} \in \Omega$, with $\pi_{i}(r) \rightarrow \infty$ as $r \rightarrow \infty$, for $i=1,2,3$;

- $\varphi: \mathbb{R}_{0}^{+} \rightarrow \mathbb{R}_{0}^{+}$, satisfying $\varphi(\cdot) \in L^{1}\left(t_{0}, \infty\right)$ and $|\varphi(\cdot)| \leq 1$;

- a $C^{1}$ functional $w(t, \psi)$ such that, for all $(t, \psi) \in\left[t_{0}, \infty\right) \times \mathcal{Q}_{A, \bar{\tau}}^{n}$,

(i) $\pi_{1}(\|\psi(0)\|) \leq w(t, \psi) \leq \pi_{2}\left(\|\psi\|_{\bar{\tau}}\right)$

(ii) along solutions to $(1 \mathrm{a}-\mathrm{b}), \dot{w}\left(t, x_{t}\right)+\pi_{3}(\|x(t)\|) \leq \varphi(t) \pi_{3}(\eta)$, where $\eta$ is a positive constant,

then solutions to (1a-c) are globally uniformly bounded and $x=0$ is globally attractive.

\section{Stabilization via unconstrained output feedback controllers}

\subsection{Class of output feedbacks}

A class of unconstrained output stabilizers is synthesized using bounding information on the uncertainties provided by Hypothesis 3 and involving some design parameters. Then, sufficient conditions for the stabilization of system (1a-c) are stated. 
The class of unconstrained output controllers, $\mathcal{F}_{u}$, consists of nonlinear functions $\tilde{f} \in \mathcal{F}_{u}$, $\tilde{f}(t, y(t)-\sigma(u(t)))=\left[\tilde{f}_{1}(t, y(t)-\sigma(u(t))), \ldots, \tilde{f}_{m}(t, y(t)-\sigma(u(t)))\right]^{\mathrm{T}}$, having the following structure, for $k \in \mathcal{J}_{m}$ :

$$
(t, y-\sigma(u)) \mapsto \tilde{f}_{k}(t, y-\sigma(u)):=-\left(1-\kappa_{k}(t)\right)^{-1}\left[\mu_{k}+\frac{\alpha_{k}(t)}{\left|r_{k}(y-\sigma(u))\right|+\varepsilon_{k}(t)}\right] r_{k}(y-\sigma(u)),
$$

where, for $k \in \mathcal{J}_{m}, \mu_{k}>0$ are design parameters, $\alpha_{k}$ and $\kappa_{k}$ are introduced in Hypothesis H3, $r_{k}$ : $\mathbb{R}^{p} \rightarrow \mathbb{R}, \varepsilon_{k}: \mathbb{R}_{0}^{+} \rightarrow(0, \epsilon]$, with $\epsilon>0$, are continuous functions satisfying $r_{k}(0)=0$ and $\varepsilon_{k}(t)>0$ for $t \in\left[t_{0}, \infty\right)$. Controller (5) does not only depend on the output $y$ but also on $\sigma(u)$, which means that the controller forms a closed-loop with itself in order to compensate the feed-through term for the stabilization. The term $\left(1-\kappa_{k}(t)\right)^{-1}$ compensates for any destabilizing effect of the input dependent term in the matched uncertainties $p_{k}, k \in \mathcal{J}_{m}$. Moreover, the term $\alpha_{k}(t) /\left[\left|r_{k}(y-\sigma(u))\right|+\varepsilon_{k}(t)\right]$ strongly reduces the impact of any offset due to $p_{k}, k \in \mathcal{J}_{m}$, on the dynamical behaviour of the system (1a-c). The parameters $\mu_{k}, k \in \mathcal{J}_{m}$, are tuning gains that act, more generally, on the other components of the uncertainty bounds, whilst their values are chosen according to the considered stability criteria, as explained later. The function $\varepsilon_{k}$ guarantees the continuity of the controller even when $r_{k}(y-\sigma(u))=0$, $k \in \mathcal{J}_{m}$ and the functions $r_{k}, k \in \mathcal{J}_{m}$, are assumed to satisfy Hypothesis H4.

H4: For all $k \in \mathcal{J}_{m}$, there exists real constants $\xi_{0, k}, \ldots, \xi_{i, k} \geq 0$, such that for all $z_{0}, \ldots, z_{i} \in \mathbb{R}^{n}$,

a) $\sum_{\gamma \in \mathcal{I}_{i}}\left\langle\nabla_{z_{\gamma}} v_{1}\left(t, z_{0}, \ldots, z_{i}\right), g_{k}\left(z_{0}\right)\right\rangle r_{k}\left(h\left(z_{0}, \ldots, z_{i}\right)\right) \geq 0$

b) $\left|r_{k}\left(h\left(z_{0}, \ldots, z_{i}\right)\right)\right| \geq\left|\sum_{\gamma \in \mathcal{I}_{i}}\left\langle\nabla_{z_{\gamma}} v_{1}\left(t, z_{0}, \ldots, z_{i}\right), g_{k}\left(z_{0}\right)\right\rangle\right|-\sum_{\gamma \in \mathcal{I}_{i}} \xi_{\gamma, k}\left\{\omega_{3}\left(\left\|z_{\gamma}\right\|\right)\right\}^{\frac{1}{2}}$

Remark 4 If the delay $\tau_{\gamma}, \gamma \in \mathcal{J}_{i}$, has no contribution to the output, then, $\forall k \in \mathcal{J}_{m}, \xi_{\gamma, k}=0$.

Remark 5 It is noted that Hypothesis $\mathrm{H}_{4} \mathrm{~b}$ ) is weaker than the corresponding hypothesis considered in Clarkson et al. [4].

Loosely speaking, an interpretation of the conditions stated in H4 is that there exist sufficient 'links' between the output function and the input matrix, and sufficient information in the output function in order that the controller is able to act significantly on the system dynamics. These conditions are quite restrictive on the types of output that are permissible; that is logical when considering the generality of the system equations being investigated. An example of system, with an output function satisfying $\mathrm{H} 4$, is given in Section 6.

\subsection{Stability analysis}

When investigating the stabilization problem for system (1a-c), utilizing the output feedback controller defined in (5), some preliminary lemmas are required, which are helpful in the sequel.

\subsubsection{Preliminary lemmas}

For notational simplicity, define $w(t):=\left[\left\{\omega_{3}(\|x(t)\|)\right\}^{\frac{1}{2}}\left\{\omega_{3}\left(\left\|x_{t}\left(-\tau_{1}(t)\right)\right\|\right)\right\}^{\frac{1}{2}} \ldots\left\{\omega_{3}\left(\left\|x_{t}\left(\tau_{i}(t)\right)\right\|\right)\right\}^{\frac{1}{2}}\right]^{\mathrm{T}}$. Lemma 1 Suppose $\mathrm{H} 2 \mathrm{e}$ ) and $\mathrm{H} 3 \mathrm{~b}$ ) hold, the following inequality is verified for all $\left(t, x_{t}\right) \in\left[t_{0}, \infty\right) \times$ $\mathcal{Q}_{A, \bar{\tau}}^{n}$,

$$
\left(D_{q} v_{1}\right)\left(t, x_{t}\right) \leq\left\langle w(t), M_{1} w(t)\right\rangle+M_{2}(t) w(t)+A w(t) \sum_{k \in \mathcal{J}_{m}} \rho_{k}\left|\left(D_{g_{k}} v_{1}\right)\left(t, x_{t}\right)\right|
$$

where

$$
\begin{aligned}
& M_{1}:=\left[\begin{array}{ccccc}
a_{0} \beta_{0} & \frac{1}{2}\left(a_{0} \beta_{1}+a_{1} \beta_{0}\right) & \ldots & \ldots & \frac{1}{2}\left(a_{0} \beta_{i}+a_{i} \beta_{0}\right) \\
\frac{1}{2}\left(a_{0} \beta_{1}+a_{1} \beta_{0}\right) & a_{1} \beta_{1} & & & \vdots \\
\vdots & & \ddots & & \vdots \\
\vdots & & & \ddots & \frac{1}{2}\left(a_{i} \beta_{i-1}+a_{i-1} \beta_{i}\right) \\
\frac{1}{2}\left(a_{0} \beta_{i}+a_{i} \beta_{0}\right) & \ldots & \ldots & \frac{1}{2}\left(a_{i} \beta_{i-1}+a_{i-1} \beta_{i}\right) & a_{i} \beta_{i}
\end{array}\right] \\
& M_{2}(t):=\alpha(t) A \text { and } A=\left[\begin{array}{llll}
a_{0} & a_{1} & \ldots & a_{i}
\end{array}\right] \text {. }
\end{aligned}
$$


Proof: This result is a straightforward consequence of $\mathrm{H} 2 \mathrm{e}$ ) and $\mathrm{H} 3 \mathrm{~b}$ ).

Lemma 2 Assuming $\mathrm{H} 3 \mathrm{a})$ and $\mathrm{H} 4$ hold and using the class of output controllers $\mathcal{F}_{u}$, defined by (5), then for all $\left(t, x(t), x_{t}, u(t)\right) \in\left[t_{0}, \infty\right) \times \mathbb{R}^{n} \times \mathcal{Q}_{A, \bar{\tau}}^{n} \times \mathcal{F}_{u}$,

$$
\begin{gathered}
A w(t) \sum_{k \in \mathcal{J}_{m}} \rho_{k}\left|\left(D_{g_{k}} v_{1}\right)\left(t, x_{t}\right)\right|+\sum_{k \in \mathcal{J}_{m}}\left[u_{k}(t)+p_{k}\left(t, x(t), x_{t}\left(-\tau_{1}(t)\right), \ldots, x_{t}\left(-\tau_{i}(t)\right), u(t)\right)\right]\left(D_{g_{k}} v_{1}\right)\left(t, x_{t}\right) \\
\leq\left\langle w(t), N_{1} w(t)\right\rangle+N_{2}(t) w(t)+\varepsilon(t),
\end{gathered}
$$

where, for $\gamma, \gamma^{\prime} \in \mathcal{I}_{i}$,

$$
N_{1}=\left[\Psi_{\gamma, \gamma^{\prime}}\left(\mu_{1}, \ldots, \mu_{m}\right)\right], \quad N_{2}(t):=\left[\begin{array}{c}
\sum_{k \in \mathcal{J}_{m}} \alpha_{k}(t) \xi_{0, k} \\
\vdots \\
\sum_{k \in \mathcal{J}_{m}} \alpha_{k}(t) \tilde{\nu}_{k}^{-1} \xi_{i, k}
\end{array}\right]^{\mathrm{T}}, \quad \varepsilon(t):=\sum_{k \in \mathcal{J}_{m}} \alpha_{k}(t) \varepsilon_{k}(t),
$$

and

$$
\begin{aligned}
\Psi_{\gamma, \gamma^{\prime}}\left(\mu_{1}, \ldots, \mu_{m}\right) & =\Psi_{\gamma^{\prime}, \gamma}\left(\mu_{1}, \ldots, \mu_{m}\right) \\
& :=\frac{1}{4} \sum_{k \in \mathcal{J}_{m}} \mu_{k}^{-1}\left(a_{\gamma} \rho_{k}+\beta_{\gamma, k}+\mu_{k} \xi_{\gamma, k}\right)\left(a_{\gamma^{\prime}} \rho_{k}+\beta_{\gamma^{\prime}, k}+\mu_{k} \xi_{\gamma^{\prime}, k}\right) .
\end{aligned}
$$

Proof: The proof of Lemma 2 is provided in Appendix A.

\subsubsection{Main theorem}

For notational convenience, consider the following matrices and constants, where $\lambda_{1}, \ldots, \lambda_{i}>0$ :

$$
R_{1}=\left[\begin{array}{cccc}
r_{0,0} & r_{0,1} & \cdots & r_{0, i} \\
r_{0,1} & r_{1,1} & \cdots & r_{1, i} \\
\vdots & \vdots & \ddots & \vdots \\
r_{0, i} & r_{1, i} & \cdots & r_{i, i}
\end{array}\right]
$$

with, for $\gamma \leq \gamma^{\prime} \in \mathcal{I}_{i}$,

$$
\begin{aligned}
r_{0,0} & =\bar{c}-\sum_{\gamma \in \mathcal{J}_{i}}\left(1-\hat{\tau}_{\gamma}\right)^{-1} \lambda_{\gamma}-a_{0} \beta_{0}-\Psi_{0,0}\left(\mu_{1}, \ldots, \mu_{m}\right), \\
r_{\gamma, \gamma} & =\lambda_{\gamma}-a_{\gamma} \beta_{\gamma}-\Psi_{\gamma, \gamma}\left(\mu_{1}, \ldots, \mu_{m}\right), \quad \text { when } \gamma \in \mathcal{J}_{i}, \\
r_{\gamma, \gamma^{\prime}} & =-\frac{1}{2}\left(a_{\gamma} \beta_{\gamma^{\prime}}+a_{\gamma^{\prime}} \beta_{\gamma}\right)-\Psi_{\gamma, \gamma^{\prime}}\left(\mu_{1}, \ldots, \mu_{m}\right)- \begin{cases}0, & \gamma=0, \\
\frac{1}{2} \bar{d}_{\left(\gamma, \gamma^{\prime}\right)}, & \text { otherwise },\end{cases}
\end{aligned}
$$

$\bar{c}:=c-\frac{1}{2} \sum_{\gamma \in \mathcal{J}_{i}}\left(1-\hat{\tau}_{\gamma}\right)^{-1} \bar{d}_{(\gamma, \gamma)}$

$$
\Upsilon_{1}\left(\lambda_{1}, \ldots, \lambda_{i}, \mu_{1}, \ldots, \mu_{m}\right)=\frac{1}{2}\left\|R_{1}^{-1}\right\|\left(\left\|R_{2}\right\|+\sqrt{\left\|R_{2}\right\|^{2}+4 \bar{\epsilon}\left\|R_{1}^{-1}\right\|}\right),
$$

$\bar{\epsilon}=\epsilon \sum_{k \in \mathcal{J}_{m}} \bar{\alpha}_{k}$, and $R_{2} \in \mathbb{R}^{1 \times i+1}$ is defined by

$$
R_{2}=\left[\begin{array}{llll}
R_{21} & 0 & \ldots & 0
\end{array}\right], \text { with } R_{21}:=\sum_{\gamma \in \mathcal{I}_{i}}\left(1-\hat{\tau}_{\gamma}\right)^{-1}\left(\hat{\alpha} a_{\gamma}+\sum_{k \in \mathcal{J}_{m}} \bar{\alpha}_{k} \xi_{\gamma, k}\right) .
$$

Invoking Lemmas 1 and 2, the following theorem can be deduced, which ensures global uniform asymptotic stability of some prescribed compact sets for system (1a-c) using the control (5). 
Theorem 4 Suppose H1-H4 hold. If there exist $\lambda_{1}, \ldots, \lambda_{i}, \mu_{1}, \ldots, \mu_{m}>0$ such that $R_{1}>0$, then, with output feedback control $u(t) \in \mathcal{F}_{u}$, defined by (5), any compact set $\mathcal{A}$, with the property $\mathcal{V}_{1}\left(\lambda_{1}, \ldots, \lambda_{i}, \mu_{1}, \ldots, \mu_{m}\right) \subsetneq \mathcal{A}$, where

$$
\mathcal{V}_{1}\left(\lambda_{1}, \ldots, \lambda_{i}, \mu_{1}, \ldots, \mu_{m}\right):=\left\{x \in \mathbb{R}^{n}:\left\{\omega_{3}(\|x\|)\right\}^{\frac{1}{2}} \leq \Upsilon_{1}\left(\lambda_{1}, \ldots, \lambda_{i}, \mu_{1}, \ldots, \mu_{m}\right)\right\}
$$

is globally uniformly asymptotically stable under the dynamics of (1a-b) subject to the initial condition (1c).

Proof: The proof of Theorem 4 is provided in Appendix B.

Remark 6 The $\lambda_{k}$ parameters are not control parameters, they are used for the Lyapunov-Krasovskii stability analysis and allow one to obtain an estimation of the sets where the states converge, when the conditions of Theorem 4 hold.

Remark 7 It is important to notice that the stability criteria of Theorem 4 require that the origin of the nominal system is globally uniformly asymptotically stable, in view of the proof in Appendix B. Indeed, matrix $R_{1}$ can be positive definite only if matrix $L_{1}$, defined in (30), is also positive definite, which implies the aforementioned stability property.

Remark 8 Although it can be seen that the stability criteria in Theorem 4 depend on the time delays, only the bounds of the time-derivatives are required. This remark also applies to all other stability criterion proposed in this study. It is clear that they become delay-independent if the time-delays are all constant.

A key question, regarding Theorem 4, is: is it always possible to find parameters $\mu_{k}$ and $\lambda_{k}$ such that $R_{1}>0$ ? In general, the answer is no, since the residual uncertainties might be too large with the result that it is impossible to compensate using the considered controllers. However, in Section 4.2.3, a sufficient analytic condition for the existence of $\mu_{k}$ and $\lambda_{k}$, such that $R_{1}>0$, is given. When solvable, numerical LMI solvers can be used in order to solve this problem. Thus, this problem can be seen as an optimisation problem under nonlinear constraints: to find $\mu_{k}$ and $\lambda_{k}$ that minimize $\Upsilon_{1}$ under the constraint $R_{1}>0$.

\subsubsection{Application of Geršgorin's theorem}

It has been shown in Section 4.2.2 that, if $R_{1}>0$, the desired stability property is ensured; here a sufficient condition that guarantees $R_{1}>0$ is given, which is derived using the Geršgorin Theorem 1 . The new condition is, obviously, more conservative than in Theorem 4 , but its resolution is much simpler and, as mentioned earlier, an analytic method for designing controller parameters $\mu_{k}$ is given.

In applying the Geršgorin Theorem 1 to the matrix $R_{1}$, the conditions, $\forall \gamma \in \mathcal{I}_{i}$,

$$
r_{\gamma, \gamma}>\sum_{\gamma^{\prime} \in \mathcal{I}_{i} \backslash\{\gamma\}}\left|r_{\gamma, \gamma^{\prime}}\right|
$$

ensure that $R_{1}>0$, which gives rise to conditions on $\lambda_{\gamma}$ :

$$
\left\{\begin{array}{l}
\forall \gamma \in \mathcal{J}_{i}, \quad \lambda_{\gamma}>\frac{1}{2} \sum_{\substack{\gamma^{\prime} \in \mathcal{J}_{i} \\
\gamma \neq \gamma^{\prime}}} \bar{d}_{\left(\gamma, \gamma^{\prime}\right)}+\frac{1}{2} \sum_{\gamma^{\prime} \in \mathcal{I}_{i}}\left(a_{\gamma} \beta_{\gamma^{\prime}}+a_{\gamma^{\prime}} \beta_{\gamma}\right)+\sum_{\gamma^{\prime} \in \mathcal{I}_{i}} \Psi_{\gamma, \gamma^{\prime}}\left(\mu_{1}, \ldots, \mu_{m}\right), \\
\bar{c}-\sum_{\gamma \in \mathcal{J}_{i}}\left(1-\hat{\tau}_{\gamma}\right)^{-1} \lambda_{\gamma}>\frac{1}{2} \sum_{\gamma^{\prime} \in \mathcal{I}_{i}}\left(a_{0} \beta_{\gamma^{\prime}}+a_{\gamma^{\prime}} \beta_{0}\right)+\sum_{\gamma^{\prime} \in \mathcal{I}_{i}} \Psi_{0, \gamma^{\prime}}\left(\mu_{1}, \ldots, \mu_{m}\right) .
\end{array}\right.
$$

Consequently, if $\bar{c}>\Gamma+\Psi\left(\mu_{1}, \ldots, \mu_{m}\right)$, where

$$
\Gamma:=\frac{1}{2} \sum_{\gamma, \gamma^{\prime} \in \mathcal{I}_{i} \times \mathcal{I}_{i}}\left(1-\hat{\tau}_{\gamma}\right)^{-1}\left(a_{\gamma} \beta_{\gamma^{\prime}}+a_{\gamma^{\prime}} \beta_{\gamma}\right)+\frac{1}{2} \sum_{\substack{\gamma, \gamma^{\prime} \in \mathcal{J}_{i} \\ \gamma \neq \gamma^{\prime}}}\left(1-\hat{\tau}_{\gamma}\right)^{-1} \bar{d}_{\left(\gamma, \gamma^{\prime}\right)}
$$


and

$$
\Psi\left(\mu_{1}, \ldots, \mu_{m}\right):=\sum_{\gamma, \gamma^{\prime} \in \mathcal{I}_{i} \times \mathcal{I}_{i}}\left(1-\hat{\tau}_{\gamma}\right)^{-1} \Psi_{\gamma, \gamma^{\prime}}\left(\mu_{1}, \ldots, \mu_{m}\right),
$$

then it is possible to select $\lambda_{\gamma}$ such that $R_{1}>0$.

Note that $\Psi\left(\mu_{1}, \ldots, \mu_{m}\right)$, which arises in Lemma 2, is important in the subsequent analysis, due to its dependence on $\mu_{k}, k \in \mathcal{J}_{m}$. To improve the stability criterion associated with the Geršgorin Theorem 1 , it is required, if possible, to minimize $\Psi$, with respect to $\mu_{k}, k \in \mathcal{J}_{m}$. Indeed, $c$ and $\Gamma$ only depend on system parameters, contrary to $\Psi$ that depends on the design parameters $\mu_{k}$. Thus, designing the control parameters $\mu_{k}$ to minimize the value of $\Psi$, the required condition is more likely to be satisfied. The term $\Psi\left(\mu_{1}, \ldots, \mu_{m}\right)$ can be decomposed into $\hat{\Psi}\left(\mu_{1}, \ldots, \mu_{m}\right)+\tilde{\Psi}\left(\mu_{1}, \ldots, \mu_{m}\right)$, where $\hat{\Psi}\left(\mu_{1}, \ldots, \mu_{m}\right)$ does not have any local minimum and $\tilde{\Psi}\left(\mu_{1}, \ldots, \mu_{m}\right)$ has a local minimum with respect to $\mu_{k}$ provided there exists $\gamma \in \mathcal{I}_{i}$ such that $\xi_{\gamma, k} \neq 0$. Consequently, introducing the set $\mathcal{K}_{m}:=\left\{k \in \mathcal{J}_{m}: \exists \gamma \in \mathcal{I}_{i}, \xi_{\gamma, k} \neq 0\right\}$, $\tilde{\Psi}\left(\mu_{1}, \ldots, \mu_{m}\right)$ and $\hat{\Psi}\left(\mu_{1}, \ldots, \mu_{m}\right)$ involve respective sums of the form $\sum_{k^{\prime} \in \mathcal{K}_{m}}(\cdot)$ and $\sum_{k^{\prime} \in \mathcal{J}_{m} \backslash \mathcal{K}_{m}}(\cdot)$. The value of $\mu_{k}, k \in \mathcal{K}_{m}$, which corresponds to a local minimum, $\tilde{\Psi}^{*}$, of $\tilde{\Psi}\left(\mu_{1}, \ldots, \mu_{m}\right)$ is denoted by $\mu_{k}=\mu_{k}^{*}$. The expression for $\mu_{k}^{*}, k \in \mathcal{K}_{m}$, is found to be

$$
\mu_{k}^{*}=\sqrt{\left(\sum_{\gamma, \gamma^{\prime} \in \mathcal{I}_{i}}\left(1-\hat{\tau}_{\gamma}\right)^{-1}\left(a_{\gamma} \rho_{k}+\beta_{\gamma, k}\right)\left(a_{\gamma^{\prime}} \rho_{k}+\beta_{\gamma^{\prime}, k}\right)\right) /\left(\sum_{\gamma, \gamma^{\prime} \in \mathcal{I}_{i}}\left(1-\hat{\tau}_{\gamma}\right)^{-1} \xi_{\gamma, k} \xi_{\gamma^{\prime}, k}\right)}
$$

and

$$
\begin{aligned}
\tilde{\Psi}^{*}: & =\tilde{\Psi}\left(\mu_{1}^{*}, \ldots, \mu_{m}^{*}\right)=\frac{1}{4} \sum_{\gamma, \gamma^{\prime} \in \mathcal{I}_{i}}\left(1-\hat{\tau}_{\gamma}\right)^{-1}\left[\xi_{\gamma, k}\left(a_{\gamma^{\prime}} \rho_{k}+\beta_{\gamma^{\prime}, k}\right)+\xi_{\gamma^{\prime}, k}\left(a_{\gamma} \rho_{k}+\beta_{\gamma, k}\right)\right] \\
& +\frac{1}{2} \sqrt{\left(\sum_{\gamma, \gamma^{\prime} \in \mathcal{I}_{i}}\left(1-\hat{\tau}_{\gamma}\right)^{-1}\left(a_{\gamma} \rho_{k}+\beta_{\gamma, k}\right)\left(a_{\gamma^{\prime}} \rho_{k}+\beta_{\gamma^{\prime}, k}\right)\right)\left(\sum_{\gamma, \gamma^{\prime} \in \mathcal{I}_{i}}\left(1-\hat{\tau}_{\gamma}\right)^{-1} \xi_{\gamma, k} \xi_{\gamma^{\prime}, k}\right)} .
\end{aligned}
$$

When $\mu_{k} \in\left\{\mu_{k}: k \in \mathcal{J}_{m} \backslash \mathcal{K}_{m}\right\}$, let $\mu_{k}^{*}$ denote the value of $\mu_{k}$ designed, sufficiently large, so that

$$
\hat{\Psi}\left(\mu_{1}, \ldots, \mu_{m}\right)<\bar{c}-\Gamma-\tilde{\Psi}^{\dagger}, \quad \text { where } \tilde{\Psi}^{\dagger}:= \begin{cases}0, & \text { if } \mathcal{K}_{m}=\emptyset \\ \tilde{\Psi}^{*}, & \text { otherwise }\end{cases}
$$

Thus, it follows that if $\bar{c}>\Gamma+\hat{\Psi}\left(\mu_{1}^{*}, \ldots, \mu_{m}^{*}\right)+\tilde{\Psi}^{\dagger}$, then there exist $\lambda_{1}, \ldots, \lambda_{i}$, satisfying (11) with, $\forall k \in \mathcal{J}_{m}, \mu_{k}=\mu_{k}^{*}$, and so $R_{1}>0$.

Theorem 5 Suppose H1-H4 hold. If $\bar{c}>\Gamma+\tilde{\Psi}^{\dagger}$, then there exist $\mu_{k}, k \in \mathcal{K}_{m}$, sufficiently large so that (16) holds and $\lambda_{1}, \ldots, \lambda_{i}$ that satisfy (11), with output feedback control $u(t)$, defined by (5), any compact set $\mathcal{A}$, with the property $\mathcal{V}_{2}\left(\lambda_{1}, \ldots, \lambda_{i}\right) \subsetneq \mathcal{A}$, where

$$
\mathcal{V}_{2}\left(\lambda_{1}, \ldots, \lambda_{i}\right):=\left\{x \in \mathbb{R}^{n}:\left\{\omega_{3}(\|x\|)\right\}^{\frac{1}{2}} \leq \Upsilon_{2}\left(\lambda_{1}, \ldots, \lambda_{i}\right)\right\}
$$

and

$$
\Upsilon_{2}\left(\lambda_{1}, \ldots, \lambda_{i}\right)=\Upsilon_{1}\left(\lambda_{1}, \ldots, \lambda_{i}, \mu_{1}^{*}, \ldots, \mu_{m}^{*}\right)
$$

is globally uniformly asymptotically stable under the dynamics of (1a-b) subject to the initial condition (1c).

Proof: The proof of Theorem 5 can be found in Appendix C. 


\subsubsection{Corollaries}

Under more restrictive conditions, it is possible to show that the set $\{0\}$ is globally attractive.

Corollary 1 Suppose H1-H4 hold. In addition, it is supposed that $\alpha \in L^{2}\left(t_{0}, \infty\right)$ and, $\forall k \in \mathcal{J}_{m}$, $\alpha_{k} \in L^{2}\left(t_{0}, \infty\right)$. If

(i) there exist $\lambda_{1}, \ldots, \lambda_{i}, \mu_{1}, \ldots, \mu_{m}>0$ such that $R_{1}>0$ holds, where $R_{1}$ is defined in (10),

$O R$

(ii) $\bar{c}>\Gamma+\tilde{\Psi}^{\dagger}$, with $\mu_{k}, k \in \mathcal{K}_{m}$, sufficiently large such that (16) holds,

then, with output feedback control $u(t) \in \mathcal{F}_{u}$, defined by $(5)$ and with $\varepsilon_{k} \in L^{2}\left(t_{0}, \infty\right)$, solutions to (1a-c) are globally uniformly bounded and $x=0$ is globally attractive.

Proof: The proof of Corollary 1 is provided in Appendix D when condition (i) applies. The case of condition (ii) follows by invoking Theorem 5 .

Remark 9 In Corollary 1, the conditions on the functions $\varepsilon_{k}$, in the controller, ensure that $\varepsilon_{k}$ are bounded, and, since $\varepsilon_{k} \in L^{2}\left(t_{0}, \infty\right)$, they converge to zero as $t \rightarrow \infty$. Often the functions $\varepsilon_{k}$ are selected to have the form $t \mapsto \varepsilon_{k}(t):=\nu e^{-\zeta t}$, where $\nu, \zeta>0$ are design parameters.

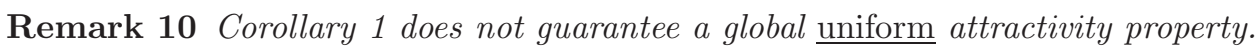

Corollary 2 Suppose H1-H4 hold, $\alpha \equiv 0$ and $\alpha_{k} \equiv 0$ for all $k \in \mathcal{J}_{m}$. If

- there exist $\lambda_{1}, \ldots, \lambda_{i}, \mu_{1}, \ldots, \mu_{m}>0$ such that $R_{1}>0$ holds, where $R_{1}$ is defined in (10),

$O R$

- $\bar{c}>\Gamma$, with $\mu_{k}, k \in \mathcal{K}_{m}$, sufficiently large such that (16) holds,

then, utilizing the output feedback control $u(t)$, defined in $(5)$, with $\varepsilon_{k} \in L^{2}\left(t_{0}, \infty\right),\{0\}$ is globally uniformly asymptotically stable for the class of systems modelled by (1a-c).

Proof: This immediately follows from Theorem 4 and Corollary 1.

\section{$5 \quad$ Stabilization via constrained output feedback controllers}

\subsection{Additional hypotheses and class of controllers}

One of the drawbacks of the controllers designed using the upper bounds to the uncertainties, as studied in Clarkson et al. [4], and $\mathrm{Wu}$ et al. [25], is that 'high gains' can be encountered, which may not satisfy the constraints on the control input of the system. Some authors have addressed the problem of constrained stabilization of functional differential equations in order to guarantee that the control input will stay within an acceptable region; for example, see Tarbouriech et al. [23] and the articles therein. In Hennet et al. [14], the problem has been addressed for known linear systems with time-delays, whilst in Clarkson et al. [4] a class of constrained memoryless state-feedback controllers has been designed to ensure global uniform asymptotic stability of some prescribed compact sets.

Nevertheless, very few researches have focused on the output stabilization of such systems. In $\mathrm{Su}$ et al. [22], delay independent control design has been proposed in which some of the conditions are relatively conservative. However in Haurani et al. [12], dynamic output controllers are designed to guarantee a local asymptotic stability property for a class of uncertain linear time-delay systems utilising some delay-dependent conditions. It is well known that delay-dependent conditions can give improved results. However, to date, there appears to be no work utilising constrained static output controllers for nonlinear uncertain time-delay systems, with a finite number of time-varying delays; this is addressed, in this section, for a given class of systems. 
It is assumed that practical conditions dictate that, for all $k \in \mathcal{J}_{m}, u_{k}$ satisfy

$$
\left|u_{k}(t)\right|<\bar{u}_{k}, \quad \forall t \geq t_{0}
$$

where $\bar{u}_{k} \in \mathbb{R}^{+}$are known.

An extra condition on the offsets of the matched uncertainties is required here, which simply ensures that the offsets of the matched uncertainties $p_{k}, k \in \mathcal{J}_{m}$, are not too big in comparison with the control input bound in order to make output control possible.

H5: For all $k \in \mathcal{J}_{m}$,

$$
\left(1-\bar{\kappa}_{k}\right)^{-1} \bar{\alpha}_{k} \leq \bar{u}_{k}
$$

The class of constrained controllers, $\mathcal{F}_{c}$, is given by $\bar{f} \in \mathcal{F}_{c}$,

$\bar{f}(t, y(t)-\sigma(u(t)))=\left[\bar{f}_{1}(t, y(t)-\sigma(u(t))), \ldots, \bar{f}_{m}(t, y(t)-\sigma(u(t)))\right]^{\mathrm{T}}$, with

$$
(t, y-\sigma(u)) \mapsto \bar{f}_{k}(t, y-\sigma(u)):=-\left(1-\kappa_{k}(t)\right)^{-1} \frac{\alpha_{k}(t)}{\left|r_{k}(y-\sigma(u))\right|+\varepsilon_{k}(t)} r_{k}(y-\sigma(u)),
$$

where $r_{k}: \mathbb{R}^{p} \rightarrow \mathbb{R}, \varepsilon_{k}: \mathbb{R}_{0}^{+} \rightarrow(0, \epsilon]$, with $\epsilon>0$, are continuous functions satisfying $r_{k}(0)=0$ and $\varepsilon_{k}(t)>0$ for $t \in\left[t_{0}, \infty\right)$. The class of controllers (18) is very similar to (5) but with no design parameter $\mu_{k}, k \in \mathcal{J}_{m}$, which may be the origin of high values in the input. Thus, assuming H5 holds, the controller (18) ensures that the constraint conditions on the input, (17), are satisfied. Concerning the functions $r_{k}, k \in \mathcal{J}_{m}$, the hypotheses in H4 are still assumed to hold, but the following hypothesis, which imposes a restrictive condition on $G(x)$, is also necessary in order to guarantee the effectiveness of the controller. H6: For all $z_{0}, \ldots, z_{i} \in \mathbb{R}^{n}$, there exist positive real constants $\chi_{\gamma, k}$, with $(\gamma, k) \in \mathcal{I}_{i} \times \mathcal{J}_{m}$, such that

$$
\left|\sum_{\gamma \in \mathcal{I}_{i}}\left\langle\nabla_{z_{\gamma}} v_{1}\left(t, z_{0}, \ldots, z_{i}\right), g_{k}\left(z_{0}\right)\right\rangle\right| \leq \sum_{\gamma \in \mathcal{I}_{i}} \chi_{\gamma, k}\left\{\omega_{3}\left(\left\|z_{\gamma}\right\|\right)\right\}^{\frac{1}{2}}
$$

\subsection{Preliminary lemmas}

In parallel with Lemma 2, the following lemma will be invoked.

Lemma 3 Assuming $\mathrm{H} 3 \mathrm{a}), \mathrm{H} 4$ and $\mathrm{H} 6$ hold, the following inequality holds for all $\left(t, x(t), x_{t}, u(t)\right) \in$ $\left[t_{0}, \infty\right) \times \mathbb{R}^{n} \times \mathcal{Q}_{A, \bar{\tau}}^{n} \times \mathcal{F}_{c}$,

$$
\begin{aligned}
A w(t) \sum_{k \in \mathcal{J}_{m}} \rho_{k}\left|\left(D_{g_{k}} v_{1}\right)\left(t, x_{t}\right)\right|+ & \sum_{k \in \mathcal{J}_{m}}\left[u_{k}(t)+p_{k}\left(t, x(t), x_{t}\left(-\tau_{1}(t)\right), \ldots, x_{t}\left(-\tau_{i}(t)\right), u(t)\right)\right]\left(D_{g_{k}} v_{1}\right)\left(t, x_{t}\right) \\
& \leq\left\langle w(t), T_{1} w(t)\right\rangle+T_{2}(t) w(t)+\varepsilon(t)
\end{aligned}
$$

where, for $\gamma, \gamma^{\prime} \in \mathcal{I}_{i}$,

$$
T_{1}=\left[\Lambda_{\gamma, \gamma^{\prime}}\right], \quad T_{2}(t)=\left[\begin{array}{c}
\sum_{k \in \mathcal{J}_{m}} \alpha_{k}(t) \xi_{0, k} \\
\vdots \\
\sum_{k \in \mathcal{J}_{m}} \alpha_{k}(t) \xi_{i, k}
\end{array}\right]^{\mathrm{T}}, \quad \varepsilon(t)=\sum_{k \in \mathcal{J}_{m}} \alpha_{k}(t) \varepsilon_{k}(t),
$$

and

$$
\Lambda_{\gamma, \gamma^{\prime}}=\Lambda_{\gamma^{\prime}, \gamma}:=\frac{1}{2} \sum_{k \in \mathcal{J}_{m}} \chi_{\gamma^{\prime}, k}\left(a_{\gamma} \rho_{k}+\beta_{\gamma, k}\right)+\frac{1}{2} \sum_{k \in \mathcal{J}_{m}} \chi_{\gamma, k}\left(a_{\gamma^{\prime}} \rho_{k}+\beta_{\gamma^{\prime}, k}\right) .
$$

Proof: The proof of Lemma 3 follows the same development as that for Lemma 2. Some specific details are provided in Appendix E. 


\subsection{Main theorems}

For presentational convenience, the following matrices and constants are introduced, with $\lambda_{1}, \ldots, \lambda_{i}>0$,

$$
\bar{R}_{1}=\left[\begin{array}{cccc}
\bar{r}_{0,0} & \bar{r}_{0,1} & \cdots & \bar{r}_{0, i} \\
\bar{r}_{0,1} & \bar{r}_{1,1} & \cdots & \bar{r}_{1, i} \\
\vdots & \vdots & \ddots & \vdots \\
\bar{r}_{0, i} & \bar{r}_{1, i} & \cdots & \bar{r}_{i, i}
\end{array}\right]
$$

with, for $\gamma \leq \gamma^{\prime} \in \mathcal{I}_{i}$,

$$
\begin{aligned}
& \bar{r}_{0,0}=\bar{c}-\sum_{\gamma \in \mathcal{J}_{i}}\left(1-\hat{\tau}_{\gamma}\right)^{-1} \lambda_{\gamma}-a_{0} \beta_{0}-\Lambda_{0,0}, \\
& \bar{r}_{\gamma, \gamma}=\lambda_{\gamma}-a_{\gamma} \beta_{\gamma}-\Lambda_{\gamma, \gamma}, \quad \text { if } \gamma \in \mathcal{J}_{i}, \\
& \bar{r}_{\gamma, \gamma^{\prime}}=-\frac{1}{2}\left(a_{\gamma} \beta_{\gamma^{\prime}}+a_{\gamma^{\prime}} \beta_{\gamma}\right)-\Lambda_{\gamma, \gamma^{\prime}}- \begin{cases}0, & \gamma=0, \\
\frac{1}{2} \bar{d}_{\left(\gamma, \gamma^{\prime}\right)}, & \text { otherwise }\end{cases}
\end{aligned}
$$

and

$$
\bar{\Upsilon}_{3}\left(\lambda_{1}, \ldots, \lambda_{i}\right):=\frac{1}{2}\left\|\bar{R}_{1}^{-1}\right\|\left(\left\|R_{2}\right\|+\sqrt{\left\|R_{2}\right\|^{2}+4 \bar{\epsilon}\left\|\bar{R}_{1}^{-1}\right\|^{-1}}\right),
$$

where $\bar{\epsilon}$ and $R_{2}$ are introduced in $\S 4.2 .2$.

In view of Lemma 3 , the following theorem may be deduced.

Theorem 6 Suppose H1-H6 hold. If there exist $\lambda_{1}, \ldots, \lambda_{i}>0$, such that $\bar{R}_{1}>0$ then, with output feedback control $u(t) \in \mathcal{F}_{c}$, defined by (18), any compact set $\mathcal{A}$, with the property $\mathcal{V}_{3}\left(\lambda_{1}, \ldots, \lambda_{i}\right) \subsetneq \mathcal{A}$, where

$$
\mathcal{V}_{3}\left(\lambda_{1}, \ldots, \lambda_{i}\right):=\left\{x \in \mathbb{R}^{n}:\left\{\omega_{3}(\|x\|)\right\}^{\frac{1}{2}} \leq \bar{\Upsilon}_{3}\left(\lambda_{1}, \ldots, \lambda_{i}\right)\right\}
$$

is globally uniformly asymptotically stable under the dynamics of (1a-b) subject to the initial condition (1c) and under the control constraints (17).

Proof: The proof of Theorem 6 is straightforward in view of Lemma 3 and the proof of Theorem 4 .

Analogous to Section 4.2.2, the existence of $\lambda_{k}$ such that $\bar{R}_{1}>0$ is not always guaranteed, thus sufficient analytic conditions for $\bar{R}_{1}>0$ are developed in Lemma 4 (see Remark 7 ).

Remark 11 Similar to Theorem 4, the condition that $\bar{R}_{1}>0$ in Theorem 6, ensures global uniform asymptotic stability of the origin of the nominal system.

Before stating the analogous theorem to Theorem 5 , the following lemma is given, that gives an explicit way to choose parameters $\lambda_{k}$ under more restrictive conditions than those in Theorem 6 . To simplify the statements of the next lemma, and following theorem and corollaries, a new notation is introduced, namely

$$
\Lambda:=\sum_{\gamma, \gamma^{\prime} \in \mathcal{I}_{i}}\left(1-\hat{\tau}_{\gamma}\right)^{-1} \Lambda_{\gamma, \gamma^{\prime}}
$$

Lemma 4 Suppose H1-H6 hold. If $\bar{c}>\Gamma+\Lambda$, where $\Gamma$ and $\Lambda$ are defined in (12) and (21), respectively, then it is possible to select, $\forall \gamma \in \mathcal{J}_{i}$,

$$
\lambda_{\gamma}>\frac{1}{2} \sum_{\substack{\gamma^{\prime} \in \mathcal{J}_{i} \\ \gamma \neq \gamma^{\prime}}} \bar{d}_{\left(\gamma, \gamma^{\prime}\right)}+\frac{1}{2} \sum_{\gamma^{\prime} \in \mathcal{I}_{i}}\left(a_{\gamma} \beta_{\gamma^{\prime}}+a_{\gamma^{\prime}} \beta_{\gamma}\right)+\sum_{\gamma \in \mathcal{I}_{i}} \Lambda_{\gamma, \gamma^{\prime}}
$$

such that $\bar{R}_{1}>0$.

Proof: This is a straightforward consequence of Geršgorin Theorem 1. Some details are provided in Appendix F. 
Consider system (1a-b) subject to control constraints (17), then the following theorem may be deduced from Theorem 6, utilising the Geršgorin Theorem 1, under the conditions specified in H1-6.

Theorem 7 Suppose H1-H6 hold. If $\bar{c}>\Gamma+\Lambda$, any compact set $\mathcal{A}$, with the property $\mathcal{V}_{4}\left(\lambda_{1}, \ldots, \lambda_{i}\right) \subsetneq$ $\mathcal{A}$, where

$$
\mathcal{V}_{4}\left(\lambda_{1}, \ldots, \lambda_{i}\right):=\left\{x \in \mathbb{R}^{n}:\left\{\omega_{3}(\|x\|)\right\}^{\frac{1}{2}} \leq \bar{\Upsilon}_{4}\left(\lambda_{1}, \ldots, \lambda_{i}\right)\right\}
$$

and

$$
\bar{\Upsilon}_{4}\left(\lambda_{1}, \ldots, \lambda_{i}\right):=\frac{1}{2}\left\|\bar{R}_{1}^{-1}\right\|\left(\left\|R_{2}\right\|+\sqrt{\left\|R_{2}\right\|^{2}+4 \bar{\epsilon}\left\|\bar{R}_{1}^{-1}\right\|^{-1}}\right)
$$

with $\lambda_{\gamma}, \gamma \in \mathcal{J}_{i}$, satisfying (22), is globally uniformly asymptotically stable for the class of systems modelled by (1a-c) with output feedback control $u(t) \in \mathcal{F}_{c}$, defined by $(18)$, when subject to the control constraints (17).

Proof: The proof of Theorem 7 is similar to the proof of Theorem 5, using Lemma 4.

\subsection{Some corollaries for the constrained case}

In parallel with Corollaries 1 and 2, the following corollaries are stated.

Corollary 3 Suppose H1-H6 hold. In addition, it is supposed that $\alpha \in L^{2}\left(t_{0}, \infty\right)$ and, $\forall k \in \mathcal{J}_{m}$, $\alpha_{k} \in L^{2}\left(t_{0}, \infty\right)$. If there exist $\lambda_{1}, \ldots, \lambda_{i}>0$ such that $\bar{R}_{1}>0$, where $\bar{R}_{1}$ is defined in (20), or if $\bar{c}>\Gamma+\Lambda$, then with output feedback control $u(t)$, subject to the control constraints (17) and defined by (18), with $\varepsilon_{k} \in L^{2}\left(t_{0}, \infty\right)$, solutions to (1a-c) are globally uniformly bounded and $x=0$ is globally attractive.

Proof: The proof of Corollary 3 is similar to that of Corollary 1 and is based on the proofs of Theorem 6 and Theorem 7.

Corollary 4 Suppose H1-H6 hold, $\alpha \equiv 0$ and $\alpha_{k} \equiv 0$ for all $k \in \mathcal{J}_{m}$. If there exist $\lambda_{\gamma}, \gamma \in \mathcal{I}_{i}$, such that $\bar{R}_{1}>0$, where $\bar{R}_{1}$ is defined in (20), or if $\bar{c}>\Gamma+\Lambda$, then utilizing the constrained output feedback control $u(t) \in \mathcal{F}_{c}$, defined in (18) with $\varepsilon_{k} \in L^{2}\left(t_{0}, \infty\right),\{0\}$ is globally uniformly asymptotically stable under the dynamics of (1a) subject to the initial condition (1c) and the control constraints (17).

Proof: This immediately follows from Theorem 6

\section{Illustrative example}

The example studied shows how an output controller of the type (18) can be used to reduce the oscillations caused by some uncertainties. Consider the system:

$$
\begin{aligned}
\dot{x}(t)= & f\left(x(t), x_{t}\left(-\tau_{1}(t)\right), x_{t}\left(-\tau_{2}(t)\right)\right)+q\left(t, x(t), x_{t}\left(-\tau_{1}(t)\right), x_{t}\left(-\tau_{2}(t)\right), u(t)\right) \\
& +G(x(t))\left[u(t)+p\left(t, x(t), x_{t}\left(-\tau_{1}(t)\right), x_{t}\left(-\tau_{2}(t)\right), u(t)\right)\right], \\
y(t)= & h\left(x(t), x_{t}\left(-\tau_{1}(t)\right), x_{t}\left(-\tau_{2}(t)\right), x_{t}\left(-\tau_{3}(t)\right)\right)+2 u(t), \\
x_{t_{0}}(\theta)= & \phi(\theta), \quad \theta \in[-\bar{\tau}, 0] \text { with } \phi(0)=x^{0},
\end{aligned}
$$

where, with $x=\left[\begin{array}{lll}x_{1} & x_{2} & x_{3}\end{array}\right]^{\mathrm{T}}$,

$$
\begin{aligned}
& f\left(x(t), x_{t}\left(-\tau_{1}(t)\right), x_{t}\left(-\tau_{2}(t)\right)\right)=A x(t)+\left[\begin{array}{c}
0 \\
0 \\
f_{1}
\end{array}\right], \quad A=\left[\begin{array}{rrr}
0 & 1 & 0 \\
0 & 0 & 1 \\
-\frac{1}{2} & -\frac{3}{2} & -1
\end{array}\right], \\
& f_{1}=\frac{1}{50}\left(x_{1}^{2}(t)+x_{2}^{2}\left(t-\tau_{2}\right)\right)^{\frac{1}{2}}+\frac{1}{80} x_{1}\left(t-\tau_{1}\right) \cos \left(x_{2}\left(t-\tau_{2}\right)\right), \quad G(x)=\left[\begin{array}{l}
0 \\
0 \\
1
\end{array}\right],
\end{aligned}
$$




$$
\begin{aligned}
h\left(x(t), x_{t}\left(-\tau_{1}(t)\right),\right. & \left.x_{t}\left(-\tau_{2}(t)\right), x_{t}\left(-\tau_{3}(t)\right)\right)=\left(x_{1}(t)+x_{2}(t)+2 x_{3}(t)\right) \psi_{1}\left(x_{t}\left(-\tau_{1}(t)\right)\right) \\
& +\Xi\left(x_{1}(t)+x_{2}(t)+2 x_{3}(t)\right)\left[\psi_{2}\left(x_{t}\left(-\tau_{2}(t)\right)\right)\left|x_{1}\left(t-\tau_{2}(t)\right)\right|+\psi_{3}\left(x_{t}\left(-\tau_{3}(t)\right)\right)\right],
\end{aligned}
$$

where $\psi_{1} \in C\left(\mathcal{Q}_{A, \bar{\tau}}^{3} ;\left[\bar{\psi}_{1}, \tilde{\psi}_{1}\right)\right)$, with $0<\bar{\psi}_{1}<\tilde{\psi}_{1}<\infty, \psi_{2} \in C\left(\mathcal{Q}_{A, \bar{\tau}}^{3} ;\left[\bar{\psi}_{2}, \tilde{\psi}_{2}\right)\right)$, with $0<\bar{\psi}_{2}<\tilde{\psi}_{2}<\infty$, $\psi_{3} \in C\left(\mathcal{Q}_{A, \bar{\tau}}^{3} ;\left[0, \tilde{\psi}_{3}\right]\right)$, with $0<\tilde{\psi}_{3}<\infty$, and $\Xi: \mathbb{R} \rightarrow \mathbb{R}, z \mapsto \Xi(z)=\Pi\left\{\begin{aligned} 1, & \text { if } z \geq 1 ; \\ z, & \text { if }|z|<1 ; \\ -1, & \text { if } z \leq-1 ;\end{aligned}\right.$ $0<\Pi<\infty$. Also, it is supposed that $K:=\bar{\psi}_{1}-\Pi \tilde{\psi}_{3}>0$.

The time-delays $\tau_{1}, \tau_{2}, \tau_{3}$ are modelled as follows: for all $t \in\left[t_{0}, \infty\right), \tau_{1}(t)=1, \tau_{2}(t)=1-\frac{1}{4} \sin ^{2}(t)$ and $\tau_{3}(t)=\frac{1}{2}$. Thus, H1 holds with $\bar{\tau}_{1}=1, \bar{\tau}_{2}=1, \bar{\tau}_{3}=\frac{1}{2}, \hat{\tau}_{1}=\hat{\tau}_{3}=0, \hat{\tau}_{2}=\frac{1}{4}$, and $\bar{\tau}=1$.

The initial conditions are selected to be $\phi(\theta)=\left[\begin{array}{lll}10 & 4 & -4\end{array}\right]^{\mathrm{T}}, \theta \in\left[\begin{array}{ll}-1,0\end{array}\right]$ and $x^{0}=\left[\begin{array}{lll}10 & 4 & -4\end{array}\right]^{\mathrm{T}}$ with $t_{0}=0$. For this system it is supposed that the control constraint $|u(t)| \leq \bar{u}=300, \forall t$, must be satisfied.

Considering the Lyapunov-Krasovskii functional $v_{1}(x):=x_{1}^{2}+\frac{3}{2} x_{2}^{2}+x_{3}^{2}+x_{1} x_{2}+x_{1} x_{3}+x_{2} x_{3}$, for $\left.x(t) \in \mathbb{R}^{3}, \mathrm{H} 2 \mathrm{a}\right)$-b) hold with: $\omega_{1}(\varpi)=\varpi^{2}$ and $\omega_{2}(\varpi)=(3+\sqrt{2}) \varpi^{2}$. A calculation shows that, along solutions to (23),

$$
\dot{v}_{1}(x(t)) \leq-s^{2}(x(t))+s_{1} s(x(t)) s\left(x\left(t-\tau_{1}\right)\right)+s_{2} s(x(t)) s\left(x\left(t-\tau_{2}\right)\right),
$$

where $s(x)=\sqrt{\hat{s}}\|x\|$, for $x \in \mathbb{R}^{3}, s_{1}=\sqrt{6} /(80 \hat{s}), s_{2}=\sqrt{6} /(50 \hat{s})$, and $\hat{s}=\frac{3}{4}-\frac{1}{4} \sqrt{5}-\frac{1}{50} \sqrt{6}$. Identifying this inequality with $(3), b_{\left(\gamma, \gamma^{\prime}\right)}=0, \forall\left(\gamma, \gamma^{\prime}\right) \in \mathcal{J}_{3} \times \mathcal{J}_{3}, \bar{d}_{(0,1)}=s_{1}, \bar{d}_{(0,2)}=s_{2}, \bar{d}_{(0,3)}=0, \bar{d}_{\left(\gamma, \gamma^{\prime}\right)}=0$ for $\left(\gamma, \gamma^{\prime}\right) \in \mathcal{J}_{3} \times \mathcal{J}_{3}$. Furthermore, it can be shown that

$$
\left(1-\sum_{\gamma \in \mathcal{J}_{3}}\left(1-\hat{\tau}_{\gamma}\right)^{-\frac{1}{2}} \bar{d}_{(0, \gamma)}\right) s^{2}(x)>0.0548\|x\|^{2}
$$

Hence, all conditions of H2c) have been verified with $c=0.0548$ and $\omega_{3}(\varpi)=\varpi^{2}$. In addition, H2 d)-e) hold with $\omega_{4}(\varpi)=\hat{s} \omega_{3}(\varpi)$, for $\varpi \in \mathbb{R}_{0}^{+}, a_{0}=3+\sqrt{2}$ and $a_{\gamma}=0$ for $\gamma \in \mathcal{J}_{3}$.

In view of Remark 2, immediately following $\mathrm{H} 2$, the trivial solution of the time-delay drift system (23), without uncertainty, is globally asymptotically uniformly stable since

$$
c>\frac{1}{2} \sum_{\left(l, l^{\prime}\right) \in \mathcal{J}_{3} \times \mathcal{J}_{3}}\left(1-\hat{\tau}_{l}\right)^{-\frac{1}{2}} \bar{d}_{\left(l, l^{\prime}\right)}=0 .
$$

The trajectories for system (23), without uncertainty, are illustrated in Figure 1.

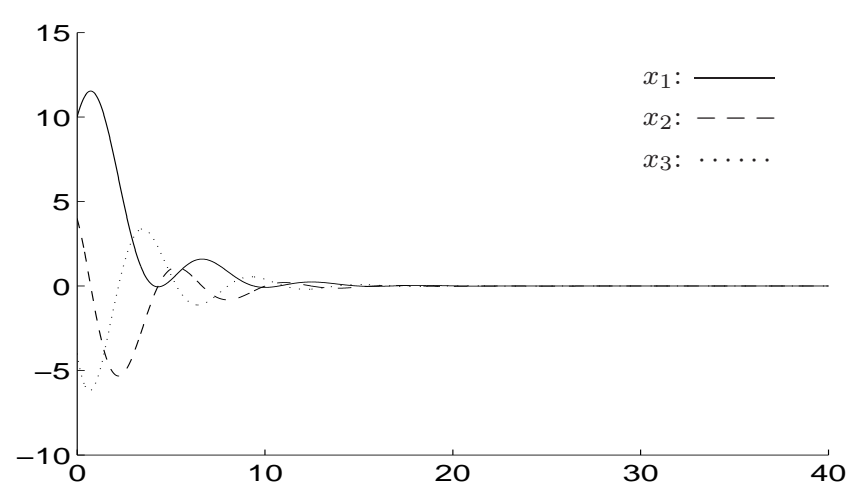

Figure 1: Open-loop response without uncertainty.

For simulation purposes, uncertainties are modelled by, $\left(t, z_{0}, z_{1}, z_{2}, z_{3}, u\right) \in\left[t_{0}, \infty\right) \times \mathbb{R}^{3} \times \mathbb{R}^{3} \times \mathbb{R}^{3} \times$ $\mathbb{R}^{3} \times \mathbb{R}$,

$$
\begin{aligned}
p\left(t, z_{0}, z_{1}, z_{2}, z_{3}, u\right) & :=\sin \left(\frac{1}{5} t\right) \eta(t)+\frac{1}{175}\left\|z_{0}\right\|+\frac{1}{120}\left(1-e^{-t}\right)\left\|z_{1}\right\|+\frac{1}{4} \sin \left(\frac{1}{4} u\right) u \\
q\left(t, z_{0}, z_{1}, z_{2}, z_{3}, u\right) & :=\left(\frac{1}{500}\left\|z_{0}\right\|+\frac{1}{500} \sin \left(\frac{1}{5} t\right)\left\|z_{2}\right\|\right)\left[\begin{array}{ccc}
0 & 1 & 0
\end{array}\right]^{\mathrm{T}} .
\end{aligned}
$$

Moreover, two cases are considered, namely
A) $\eta(t)=200 t^{2} /\left(20+t^{2}\right)$ and $\varepsilon_{1}(t)=0.01$;
B) $\eta(t)=48 t e^{-t / 12}$ and $\varepsilon_{1}(t)=e^{-t / 9}$, 
which will illustrate the results of Theorem 7 and Corollary 3 . Note that the conditions of $\mathrm{H} 3$ are satisfied with: $\alpha_{1}(t)=\left|\sin \left(\frac{1}{5} t\right)\right| \eta(t), \beta_{0,1}=\frac{1}{175}, \beta_{1,1}=\frac{1}{120}, \beta_{2,1}=\beta_{3,1}=0, \kappa_{1}(t)=\bar{\kappa}_{1}=\frac{1}{4}$ for all $t$, $\alpha(t) \equiv 0, \beta_{0}=\beta_{2}=\frac{1}{500}, \beta_{1}=\beta_{3}=0$, and $\rho_{1}=0$. The open-loop response, with uncertainty, for both cases is presented in Figure 2 .

A)

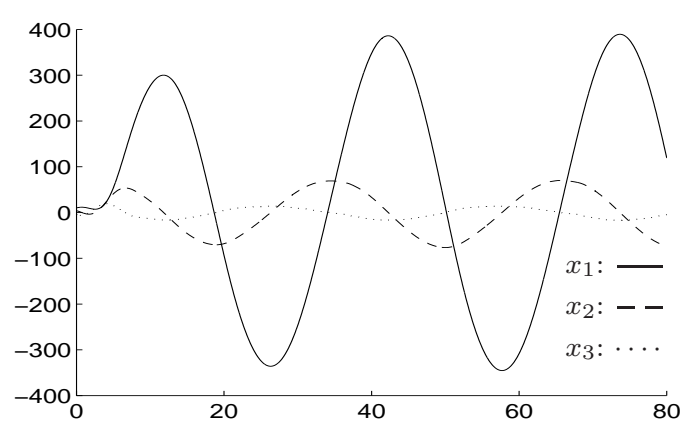

B)

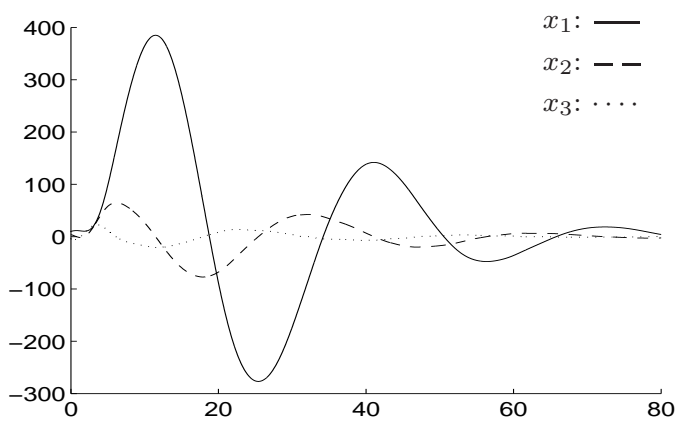

Figure 2: Open-loop response, with uncertainty for A) $\eta(t)=200 t^{2} /\left(20+t^{2}\right)$; B) $\eta(t)=48 t e^{-t / 12}$.

The matched and residual uncertainty, namely $p$ and $q$, for the open-loop case are illustrated in Figure 3 , for case A); and Figure 4, for case B).

(i)

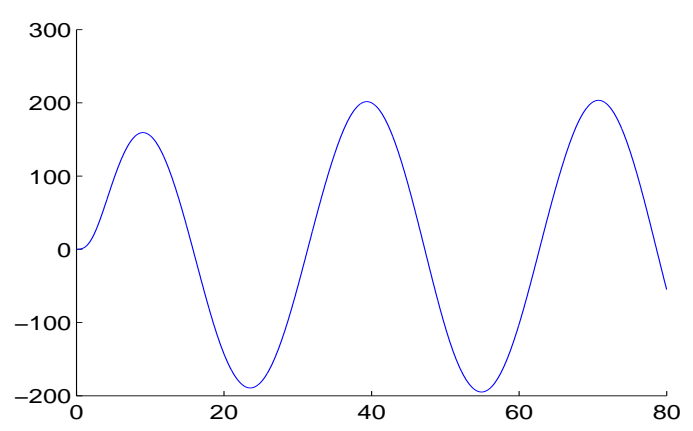

Figure 3: Case A): (i) Matched uncertainty; (ii) Residual uncertainty. (ii)

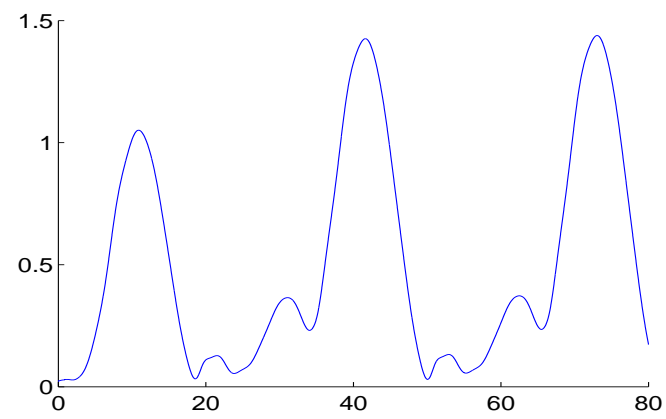

(i)

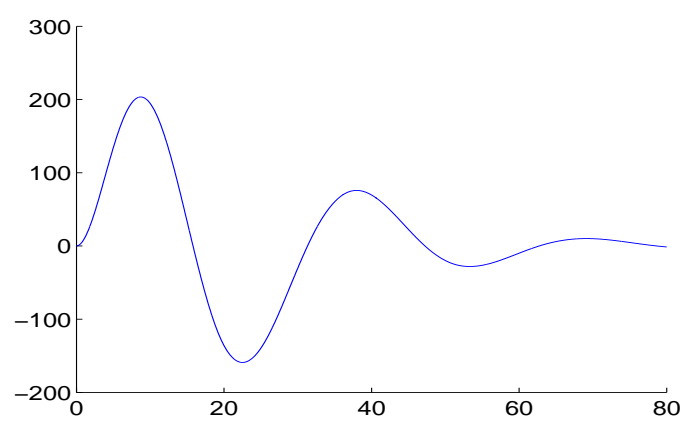

(ii)

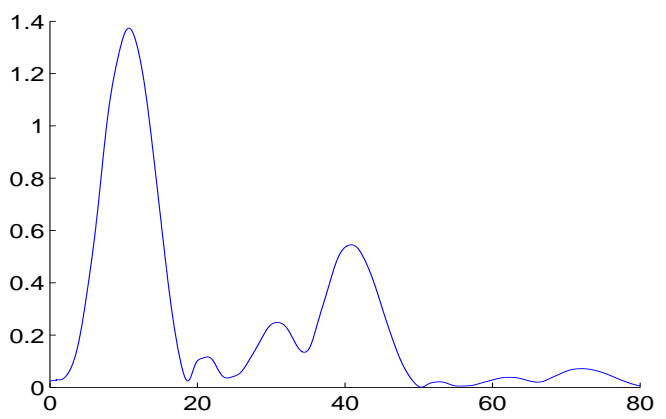

Figure 4: Case B): (i) Matched uncertainty; (ii) Residual uncertainty.

For the constrained output controller, the function $r$ is chosen to be $r: a \mapsto a / K$, for $a \in \mathbb{R}$. In this case,

$$
r_{1}\left(h\left(z_{0}, z_{1}, z_{2}, z_{3}\right)\right)=K^{-1}\left(Y \psi_{1}\left(z_{1}\right)+\Xi(Y)\left[\psi_{2}\left(z_{2}\right)\left|z_{2}^{(1)}\right|+\psi_{3}\left(z_{3}\right)\right]\right),
$$


where $Y=z_{0}^{(1)}+z_{0}^{(2)}+2 z_{0}^{(3)}, z_{0}=\left[\begin{array}{lll}z_{0}^{(1)} & z_{0}^{(2)} & z_{0}^{(3)}\end{array}\right]^{\mathrm{T}}$, and $z_{2}=\left[\begin{array}{lll}z_{2}^{(1)} & z_{2}^{(2)} & z_{2}^{(3)}\end{array}\right]^{\mathrm{T}}$. Therefore, since $g_{1}\left(z_{0}\right)=\left[\begin{array}{lll}0 & 0 & 1\end{array}\right]^{\mathrm{T}}$ and, $\forall z \in \mathbb{R}, z \Xi(z) \geq 0$, for all $\left(z_{0}, z_{1}, z_{2}, z_{3}\right)$ it follows that

$$
\left\langle\nabla_{z_{0}} v_{1}\left(z_{0}\right), g_{1}\left(z_{0}\right)\right\rangle=K^{-1}\left(Y^{2} \psi_{1}\left(z_{1}\right)+Y \Xi(Y)\left[\psi_{2}\left(z_{2}\right)\left|z_{2}^{(1)}\right|+\psi_{3}\left(z_{3}\right)\right]\right) \geq 0 .
$$

Hence, the condition specified in H4a) holds. Now consider the inequality in H4b). Using (26) and since, $\forall z \in \mathbb{R},|\Xi(z)| \leq \Pi$ and $|\Xi(z)| \leq \Pi|z|$,

$$
\bar{\psi}_{1}|Y| \leq K \mid r_{1}\left(h\left(z_{0}, z_{1}, z_{2}, z_{3}\right)|+| \Xi(Y) \mid\left[\tilde{\psi}_{2}\left\|z_{2}\right\|+\tilde{\psi}_{3}\right]\right.
$$

and so

$$
K \mid r_{1}\left(h\left(z_{0}, z_{1}, z_{2}, z_{3}\right)\left|\geq\left(\bar{\psi}_{1}-\Pi \tilde{\psi}_{3}\right)\right| Y \mid-\Pi \tilde{\psi}_{2}\left\|z_{2}\right\| .\right.
$$

Therefore H4b) is satisfied with $\xi_{0,1}=\xi_{1,1}=\xi_{3,1}=0$ and $\xi_{2,1}=\Pi \tilde{\psi}_{2} / K$. For simulation, with $z=\left[\begin{array}{lll}z^{(1)} & z^{(2)} & z^{(3)}\end{array}\right]$, let $\psi_{1}(z)=4+3 \sin ^{2}\left(z^{(1)}\right), \psi_{2}(z) \equiv 1, \psi_{3}(z)=\left[z^{(1)}\right]^{2} /\left(1+\left[z^{(1)}\right]^{2}\right)$ and $\Pi=2$ so that $\bar{\psi}_{1}=4, \tilde{\psi}_{2}=1, \tilde{\psi}_{3}=1$, and, therefore, $K=2$ and $\xi_{2,1}=1$.

Considering the upper bound of the control input fixed at $\bar{u}=300$, for both cases $\mathrm{A}$ ) and $\mathrm{B}$ ), $\bar{\alpha}_{1}=200$ and so H5 is satisfied, since $\left(1-\bar{\kappa}_{1}\right)^{-1} \alpha_{1}=\frac{800}{3}<\bar{u}$. Moreover, H6 holds with $\chi_{0,1}=\sqrt{6}$, and $\chi_{1,1}=\chi_{2,1}=\chi_{3,1}=0$.

Only Theorem 7 and Corollary 3 are applied here. Calculations show that $\Gamma<0.01913$ and $\Lambda<$ 0.03441 , and so $\Gamma+\Lambda<0.05354<\bar{c}=c=0.0548$. Consequently, for case A) and utilising Theorem 7 , the controller:

$$
-\frac{800 t^{2}|\sin (t / 5)|}{3\left(20+t^{2}\right)}\left[\frac{0.5(y(t)-2 u(t))}{0.5|y(t)-2 u(t)|+\varepsilon_{1}(t)}\right]
$$

with $\varepsilon_{1}(t)=0.01$ for all $t$, ensures the global uniform asymptotic stability of a compact set, whilst, for case B) and utilising Corollary 3, the controller:

$$
-64 t e^{-t / 12}|\sin (t / 5)|\left[\frac{0.5(y(t)-2 u(t))}{0.5|y(t)-2 u(t)|+\varepsilon_{1}(t)}\right],
$$

with $\varepsilon_{1}(t)=e^{-t / 9}$ for all $t$, ensures that $x=0$ is globally attractive.

The closed-loop response and control history for case A) are shown in Figure 5, whilst those for case B) are illustrated in Figure 6. It is clearly seen from the simulations that the control inputs satisfy the condition $u<\bar{u}=300$, as shown in Figures 5 (ii) and 6 (ii).

(i)

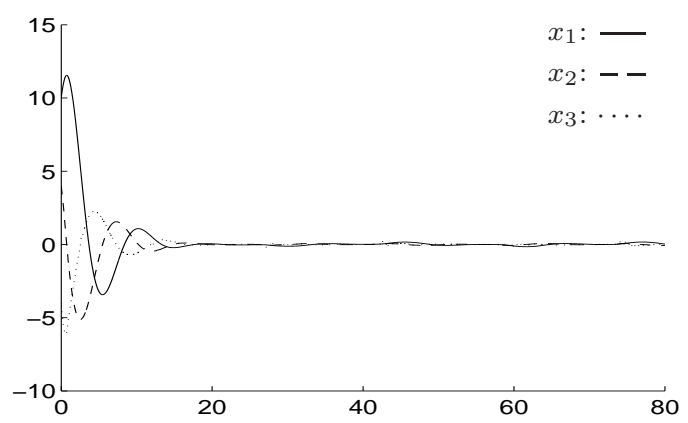

(ii)

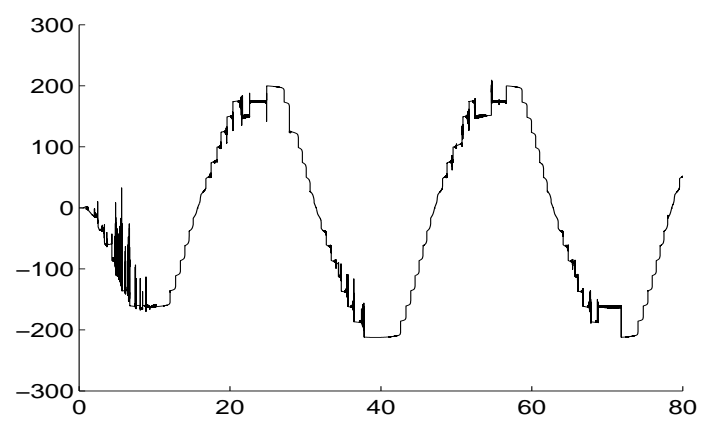

Figure 5: Case A): (i) Closed-loop response; (ii) control history.

The output histories, for cases A) and B), are shown in Figure 7.

\section{Conclusion}

In this study, a class of robust static output controllers for the stabilization of a class of imperfectly known nonlinear dynamical systems, affected by time-varying delays, has been synthesized, where the 
(i)

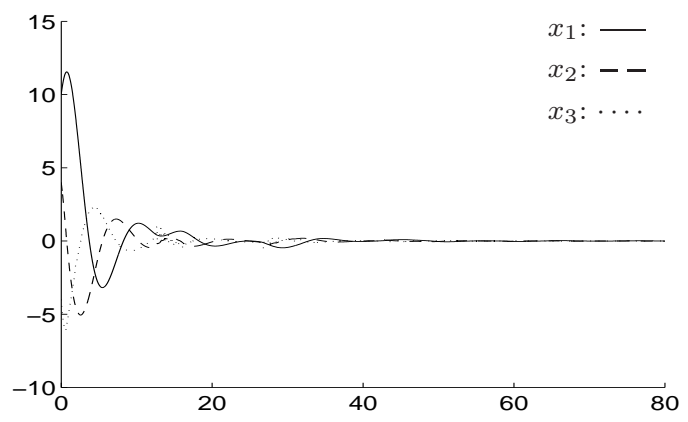

(ii)

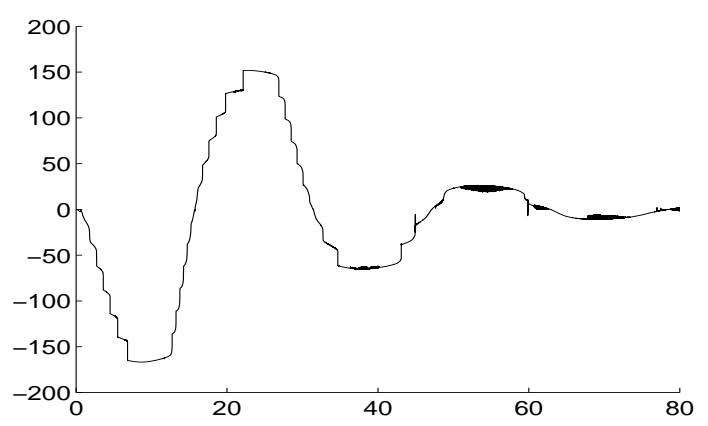

Figure 6: Case B): (i) Closed-loop response; (ii) control history.

A)

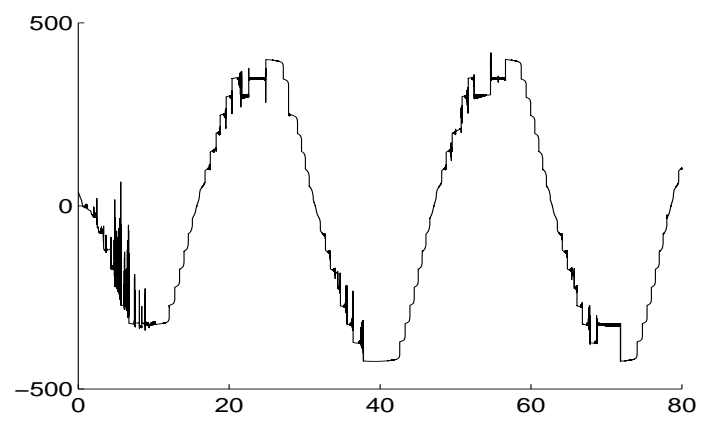

B)

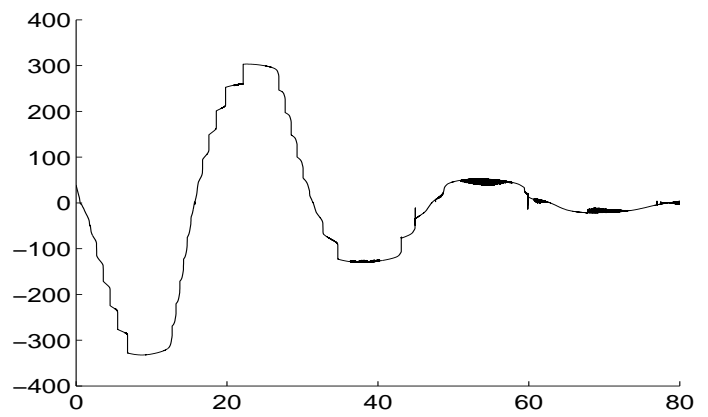

Figure 7: Output histories for cases A) and B).

output of the system is modelled by a nonlinear function, which may depend on the delayed states, and inputs, together with a feed-through term. Moreover, bounding information on the perturbations is assumed to be known in terms of specified growth conditions; it is not necessary that the values of the perturbations lie in some compact set. Sufficient conditions for the stabilization have been stated, which are an improvement of those presented in the work of Clarkson et al. [3]. Moreover, both unconstrained and constrained output stabilization have been investigated.

\section{Appendix A Proof of Lemma 2}

Using H3 a), H4 a) and in view of (5), for all $\left(t, x(t), x_{t}, u(t)\right) \in\left[t_{0}, \infty\right) \times \mathbb{R}^{n} \times \mathcal{Q}_{A, \bar{\tau}}^{n} \times \mathbb{R}^{m}$,

$$
\begin{aligned}
{\left[u_{k}(t)+\right.} & \left.p_{k}\left(t, x(t), x_{t}\left(-\tau_{1}(t)\right), \ldots, x_{t}\left(-\tau_{i}(t)\right), u(t)\right)\right]\left(D_{g_{k}} v_{1}\right)\left(t, x_{t}\right) \\
\leq & {\left[\alpha_{k}(t)+\sum_{\gamma \in \mathcal{I}_{i}} \beta_{\gamma, k}\left\{\omega_{3}\left(\left\|x_{t}\left(-\tau_{\gamma}(t)\right)\right\|\right)\right\}^{\frac{1}{2}}\right.} \\
& \left.\quad-\left(\mu_{k}+\frac{\alpha_{k}(t)}{\left|r_{k}(y(t)-\sigma(u(t)))\right|+\varepsilon_{k}(t)}\right)\left|r_{k}(y(t)-\sigma(u(t)))\right|\right]\left|\left(D_{g_{k}} v_{1}\right)\left(t, x_{t}\right)\right| .
\end{aligned}
$$


In view of $\mathrm{H} 4 \mathrm{~b})$,

$$
\begin{aligned}
{\left[\alpha_{k}(t)\right.} & \left.-\frac{\alpha_{k}(t)\left|r_{k}(y(t)-\sigma(u(t)))\right|}{\left|r_{k}(y(t)-\sigma(u(t)))\right|+\varepsilon_{k}(t)}\right]\left|\left(D_{g_{k}} v_{1}\right)\left(t, x_{t}\right)\right| \\
& =\frac{\varepsilon_{k}(t) \alpha_{k}(t)\left|\left(D_{g_{k}} v_{1}\right)\left(t, x_{t}\right)\right|}{\left|r_{k}(y(t)-\sigma(u(t)))\right|+\varepsilon_{k}(t)} \\
& \leq \frac{\alpha_{k}(t) \varepsilon_{k}(t)\left[\left|r_{k}(y(t)-\sigma(u(t)))\right|+\sum_{\gamma \in \mathcal{I}_{i}} \xi_{\gamma, k}\left\{\omega_{3}\left(\left\|x_{t}\left(-\tau_{\gamma}(t)\right)\right\|\right)\right\}^{\frac{1}{2}}\right]}{\left|r_{k}(y(t)-\sigma(u(t)))\right|+\varepsilon_{k}(t)} \\
& \leq \alpha_{k}(t) \varepsilon_{k}(t)+\alpha_{k}(t) \sum_{\gamma \in \mathcal{I}_{i}} \xi_{\gamma, k}\left\{\omega_{3}\left(\left\|x_{t}\left(-\tau_{\gamma}(t)\right)\right\|\right)\right\}^{\frac{1}{2}}
\end{aligned}
$$

and so

$$
\begin{aligned}
{\left[u_{k}(t)+\right.} & \left.p_{k}\left(t, x(t), x_{t}\left(-\tau_{1}(t)\right), \ldots, x_{t}\left(-\tau_{i}(t)\right), u(t)\right)\right]\left(D_{g_{k}} v_{1}\right)\left(t, x_{t}\right) \\
\leq & \left|\left(D_{g_{k}} v_{1}\right)\left(t, x_{t}\right)\right|\left[-\mu_{k}\left|r_{k}(y(t)-\sigma(u(t)))\right|+\sum_{\gamma \in \mathcal{I}_{i}} \beta_{\gamma, k}\left\{\omega_{3}\left(\left\|x_{t}\left(-\tau_{\gamma}(t)\right)\right\|\right)\right\}^{\frac{1}{2}}\right] \\
& +\alpha_{k}(t) \varepsilon_{k}(t)+\alpha_{k}(t) \sum_{\gamma \in \mathcal{I}_{i}} \xi_{\gamma, k}\left\{\omega_{3}\left(\left\|x_{t}\left(-\tau_{\gamma}(t)\right)\right\|\right)\right\}^{\frac{1}{2}} .
\end{aligned}
$$

Applying $\mathrm{H} 4 \mathrm{~b}$ ),

$$
\begin{aligned}
-\mu_{k}\left|r_{k}(y(t)-\sigma(u(t)))\right|\left|\left(D_{g_{k}} v_{1}\right)\left(t, x_{t}\right)\right| \leq & -\mu_{k}\left|\left(D_{g_{k}} v_{1}\right)\left(t, x_{t}\right)\right|^{2} \\
& +\mu_{k}\left|\left(D_{g_{k}} v_{1}\right)\left(t, x_{t}\right)\right| \sum_{\gamma \in \mathcal{I}_{i}} \xi_{\gamma, k}\left\{\omega_{3}\left(\left\|x_{t}\left(-\tau_{\gamma}(t)\right)\right\|\right)\right\}^{\frac{1}{2}}
\end{aligned}
$$

and so it follows, from (27), that

$$
\begin{aligned}
{\left[u_{k}(t)+\right.} & \left.p_{k}\left(t, x(t), x_{t}\left(-\tau_{1}(t)\right), \ldots, x_{t}\left(-\tau_{i}(t)\right), u(t)\right)\right]\left(D_{g_{k}} v_{1}\right)\left(t, x_{t}\right) \\
\leq & -\mu_{k}\left|\left(D_{g_{k}} v_{1}\right)\left(t, x_{t}\right)\right|^{2}+\left|\left(D_{g_{k}} v_{1}\right)\left(t, x_{t}\right)\right| \sum_{\gamma \in \mathcal{I}_{i}}\left(\beta_{\gamma, k}+\mu_{k} \xi_{\gamma, k}\right)\left\{\omega_{3}\left(\left\|x_{t}\left(-\tau_{\gamma}(t)\right)\right\|\right)\right\}^{\frac{1}{2}} \\
& +\alpha_{k}(t) \varepsilon_{k}(t)+\alpha_{k}(t) \sum_{\gamma \in \mathcal{I}_{i}} \xi_{\gamma, k}\left\{\omega_{3}\left(\left\|x_{t}\left(-\tau_{\gamma}(t)\right)\right\|\right)\right\}^{\frac{1}{2}} .
\end{aligned}
$$

Hence,

$$
\begin{aligned}
& A w(t) \rho_{k}\left|\left(D_{g_{k}} v_{1}\right)\left(t, x_{t}\right)\right|+\left[u_{k}(t)+p_{k}\left(t, x(t), x_{t}\left(-\tau_{1}(t)\right), \ldots, x_{t}\left(-\tau_{i}(t)\right), u(t)\right)\right]\left(D_{g_{k}} v_{1}\right)\left(t, x_{t}\right) \\
& \leq-\mu_{k}\left|\left(D_{g_{k}} v_{1}\right)\left(t, x_{t}\right)\right|^{2}+\left|\left(D_{g_{k}} v_{1}\right)\left(t, x_{t}\right)\right| \sum_{\gamma \in \mathcal{I}_{i}}\left(a_{\gamma} \rho_{k}+\beta_{\gamma, k}+\mu_{k} \xi_{\gamma, k}\right)\left\{\omega_{3}\left(\left\|x_{t}\left(-\tau_{\gamma}(t)\right)\right\|\right)\right\}^{\frac{1}{2}} \\
& \quad+\alpha_{k}(t) \varepsilon_{k}(t)+\alpha_{k}(t) \sum_{\gamma \in \mathcal{I}_{i}} \xi_{\gamma, k}\left\{\omega_{3}\left(\left\|x_{t}\left(-\tau_{\gamma}(t)\right)\right\|\right)\right\}^{\frac{1}{2}} .
\end{aligned}
$$

Using the inequality $-a u^{2}+u v \leq v^{2} /(4 a)$, for $a \in \mathbb{R} \backslash\{0\}$ and $(u, v) \in \mathbb{R}^{2}$,

$$
\begin{aligned}
& -\mu_{k}\left|\left(D_{g_{k}} v_{1}\right)\left(t, x_{t}\right)\right|^{2}+\left|\left(D_{g_{k}} v_{1}\right)\left(t, x_{t}\right)\right| \sum_{\gamma \in \mathcal{I}_{i}}\left(a_{\gamma} \rho_{k}+\beta_{\gamma, k}+\mu_{k} \xi_{\gamma, k}\right)\left\{\omega_{3}\left(\left\|x_{t}\left(-\tau_{\gamma}(t)\right)\right\|\right)\right\}^{\frac{1}{2}} \\
& \leq \frac{1}{4 \mu_{k}}\left[\sum_{\gamma \in \mathcal{I}_{i}}\left(a_{\gamma} \rho_{k}+\beta_{\gamma, k}+\mu_{k} \xi_{\gamma, k}\right)\left\{\omega_{3}\left(\left\|x_{t}\left(-\tau_{\gamma}(t)\right)\right\|\right)\right\}^{\frac{1}{2}}\right]^{2} .
\end{aligned}
$$

and so, from (28),

$$
\begin{aligned}
& A w(t) \rho_{k}\left|\left(D_{g_{k}} v_{1}\right)\left(t, x_{t}\right)\right|+\left[u_{k}(t)+p_{k}\left(t, x(t), x_{t}\left(-\tau_{1}(t)\right), \ldots, x_{t}\left(-\tau_{i}(t)\right), u(t)\right)\right]\left(D_{g_{k}} v_{1}\right)\left(t, x_{t}\right) \\
& \leq \frac{1}{4 \mu_{k}}\left[\sum_{\gamma \in \mathcal{I}_{i}}\left(a_{\gamma} \rho_{k}+\beta_{\gamma, k}+\mu_{k} \xi_{\gamma, k}\right)\left\{\omega_{3}\left(\left\|x_{t}\left(-\tau_{\gamma}(t)\right)\right\|\right)\right\}^{\frac{1}{2}}\right]^{2} \\
& \quad+\alpha_{k}(t) \varepsilon_{k}(t)+\alpha_{k}(t) \sum_{\gamma \in \mathcal{I}_{i}} \xi_{\gamma, k}\left\{\omega_{3}\left(\left\|x_{t}\left(-\tau_{\gamma}(t)\right)\right\|\right)\right\}^{\frac{1}{2}} .
\end{aligned}
$$

This immediately produces the required result. 


\section{Appendix B Proof of Theorem 4}

The proof consists in checking conditions (i) and (ii) of Theorem 2.

\section{Condition (i) of Theorem 2}

Consider the Lyapunov-Krasovskii functional, for $(t, \psi) \in\left[t_{0}, \infty\right) \times \mathcal{Q}_{A, \bar{\tau}}^{n}$,

$$
v(t, \psi):=v_{1}\left(t, \psi(0), \psi\left(-\tau_{1}(t)\right), \ldots \psi\left(-\tau_{i}(t)\right)\right)+v_{2}(t, \psi)+v_{3}(t, \psi)+v_{4}(t, \psi)+v_{5}(t, \psi),
$$

with $v_{1}$ introduced in $\mathrm{H} 2$,

$$
\begin{aligned}
& v_{2}(t, \psi)=\sum_{\gamma \in \mathcal{J}_{i}} \int_{-\tau_{\gamma}(t)}^{0}\left(1-\hat{\tau}_{\gamma}\right)^{-1} \varrho_{\gamma} s^{2}(\psi(\theta)) \mathrm{d} \theta, \\
& v_{3}(t, \psi)=\sum_{\left(\gamma, \gamma^{\prime}\right) \in \mathcal{J}_{i} \times \mathcal{J}_{n}} \int_{-\tau_{\gamma}(t)}^{0}\left(1-\hat{\tau}_{\gamma}\right)^{-1} b_{\left(\gamma, \gamma^{\prime}\right)} \omega_{\gamma+4}\left(\left|\psi_{\gamma^{\prime}}(\theta)\right|\right) \mathrm{d} \theta, \\
& v_{4}(t, \psi)=\sum_{\gamma \in \mathcal{J}_{i}} \int_{-\tau_{\gamma}(t)}^{0}\left(1-\hat{\tau}_{\gamma}\right)^{-1}\left(\lambda_{\gamma}+\frac{1}{2} \bar{d}_{(\gamma, \gamma)}\right) \omega_{3}(\|\psi(\theta)\|) \mathrm{d} \theta, \\
& v_{5}(t, \psi)=\sum_{\gamma \in \mathcal{J}_{i}} \int_{-\tau_{\gamma}(t)}^{0}\left(1-\hat{\tau}_{\gamma}\right)^{-1} \varsigma_{\gamma}\left\{\omega_{3}(\|\psi(\theta)\|)\right\}^{\frac{1}{2}} \mathrm{~d} \theta,
\end{aligned}
$$

where $\varrho_{\gamma}, \lambda_{\gamma}, \varsigma_{\gamma} \geq 0, \gamma \in \mathcal{J}_{i}$, are parameters with $\varrho_{\gamma}, \lambda_{\gamma}$ and $\varsigma_{\gamma}$ specified later. Clearly, by definition of $v$ (see (29)) and in view of $\mathrm{H} 2 \mathrm{~b}$ ), the functional $v$ satisfies $v(t, \psi) \geq v_{1}\left(t, \psi(0), \psi\left(-\tau_{1}(t)\right), \ldots \psi\left(-\tau_{i}(t)\right)\right) \geq$ $\omega_{1}(\|\psi(0)\|)$. Also, since, for all $\left(\gamma, \gamma^{\prime}\right) \in \mathcal{J}_{i} \times \mathcal{J}_{n}, \exists \omega_{\gamma+4}^{*} \in \Omega$ such that, for all $\psi=\left[\psi_{1} \ldots \psi_{n}\right]^{\mathrm{T}} \in \mathcal{Q}_{A, \bar{\tau}}^{n}$, $\omega_{\gamma+4}\left(\left|\psi_{\gamma^{\prime}}\right|_{\bar{\tau}}\right) \leq \omega_{\gamma+4}^{*}\left(\|\psi\|_{\bar{\tau}}\right)$, and in view of $\left.\mathrm{H} 2 \mathrm{~b}\right)$ and $\left.\mathrm{H} 2 \mathrm{~d}\right)$, standard arguments show that

$$
\begin{aligned}
v(t, \psi) \leq \omega_{2}\left(\|\psi(\theta)\|_{\bar{\tau}}+\sum_{\left(\gamma, \gamma^{\prime}\right) \in \mathcal{J}_{i} \times \mathcal{J}_{n}} \bar{\tau}_{\gamma}\left(1-\hat{\tau}_{\gamma}\right)^{-1}\left[\varrho_{\gamma} \omega_{4}\left(\|\psi(\theta)\|_{\bar{\tau}}\right)+b_{\left(\gamma, \gamma^{\prime}\right)} \omega_{\gamma+4}^{*}\left(\|\psi(\theta)\|_{\bar{\tau}}\right)\right.\right. \\
\left.+\left(\lambda_{\gamma}+\frac{1}{2} \bar{d}_{(\gamma, \gamma)}\right) \omega_{3}\left(\|\psi(\theta)\|_{\bar{\tau}}\right)+\varsigma_{\gamma}\left\{\omega_{3}\left(\|\psi(\theta)\|_{\bar{\tau}}\right)\right\}^{\frac{1}{2}}\right] .
\end{aligned}
$$

Thus, there exist $\pi_{1}, \pi_{2} \in \Omega$ such that $\pi_{1}(\|\psi(0)\|) \leq v(t, \psi) \leq \pi_{2}\left(\|\psi\|_{\bar{\tau}}\right)$ and, hence, condition (i) of Theorem 2 is valid.

\section{Condition (ii) of Theorem 2}

Firstly, it is shown that solutions to (1a-c) exist and can be continued indefinitely. By Theorem 2.7.11, $\S 2.7$, Chapter 2 of Michel et al [17], there exist a local solution to system (1a-c). According to Theorem $2.7 .15, \S 2.7$, Chapter 2 of Michel et al [17], if it can be shown that all solutions to (1a-c) are bounded, then every solution can be extended to the interval $\left[t_{0}, \infty\right)$. Utilising $\mathrm{H} 2 \mathrm{c}$ ), the time-derivatives of $v_{2}$ and $v_{3}$, and using the inequality $-a u^{2}+u v \leq v^{2} /(4 a)$ for $a \in \mathbb{R} \backslash\{0\}$ and $(u, v) \in \mathbb{R}^{2}$, along every solution of (1a-b), the following inequality can be shown to hold:

$$
\begin{aligned}
\dot{v}_{1}\left(t, x_{t}\right) & +\dot{v}_{2}\left(t, x_{t}\right)+\dot{v}_{3}\left(t, x_{t}\right) \leq-s^{2}(x(t))+\sum_{\left(\gamma, \gamma^{\prime}\right) \in \mathcal{J}_{i} \times \mathcal{J}_{n}}\left(1-\hat{\tau}_{\gamma}\right)^{-1} b_{\left(\gamma, \gamma^{\prime}\right)} \omega_{\gamma+4}\left(\left|x_{\gamma^{\prime}}(t)\right|\right) \\
& +\frac{1}{2} \sum_{\left(l, l^{\prime}\right) \in \mathcal{J}_{i} \times \mathcal{J}_{i}} \bar{d}_{\left(l, l^{\prime}\right)}\left\{\omega_{3}\left(\left\|x_{t}\left(-\tau_{l}(t)\right)\right\|\right) \omega_{3}\left(\left\|x_{t}\left(-\tau_{l^{\prime}}(t)\right)\right\|\right)\right\}^{\frac{1}{2}} \\
& +\sum_{\gamma \in \mathcal{J}_{i}}\left(1-\hat{\tau}_{\gamma}\right)^{-1} \varrho_{\gamma} s^{2}(x(t))+\frac{1}{4} \sum_{\gamma \in \mathcal{J}_{i}} \varrho_{\gamma}^{-1} \bar{d}_{(0, \gamma)}^{2} s^{2}(x(t)) \\
& +\sum_{k \in \mathcal{J}_{m}}\left[u_{k}(t)+p_{k}\left(t, x(t), x_{t}\left(-\tau_{1}(t)\right), \ldots, x_{t}\left(-\tau_{i}(t)\right), u(t)\right)\right]\left(D_{g_{k}} v_{1}\right)\left(t, x_{t}\right)+\left(D_{q} v_{1}\right)\left(t, x_{t}\right),
\end{aligned}
$$


almost everywhere. Moreover, because the function $a \mapsto c_{1} a+c_{2} a^{-1}$, with $\left(c_{1}, c_{2}\right) \in \mathbb{R}^{+} \times \mathbb{R}^{+}$, has a local minimum $2 \sqrt{c_{1} c_{2}}$, in the region $a>0$ when $a=\sqrt{\frac{c_{2}}{c_{1}}}$, then, for all $\gamma \in \mathcal{J}_{i},\left(1-\hat{\tau}_{\gamma}\right)^{-1} \varrho_{\gamma}+\frac{1}{4} \varrho_{\gamma}^{-1} \bar{d}_{0, \gamma}^{2}$ has a local minimum $\left(1-\hat{\tau}_{\gamma}\right)^{-\frac{1}{2}} \bar{d}_{(0, \gamma)}$ when $\varrho_{\gamma}=\frac{1}{2}\left(1-\hat{\tau}_{\gamma}\right)^{\frac{1}{2}} \bar{d}_{(0, \gamma)}$. Thus, along solutions to (1a-b) and with $\varrho_{\gamma}=\frac{1}{2}\left(1-\hat{\tau}_{\gamma}\right)^{\frac{1}{2}} \bar{d}_{(0, \gamma)}$ for all $\gamma \in \mathcal{J}_{i}$,

$$
\begin{aligned}
\dot{v}_{1}\left(t, x_{t}\right) & +\dot{v}_{2}\left(t, x_{t}\right)+\dot{v}_{3}\left(t, x_{t}\right) \leq-\left(1-\sum_{\gamma \in \mathcal{J}_{i}}\left(1-\hat{\tau}_{\gamma}\right)^{-\frac{1}{2}} \bar{d}_{(0, \gamma)}\right) s^{2}(x(t)) \\
& +\sum_{\left(\gamma, \gamma^{\prime}\right) \in \mathcal{J}_{i} \times \mathcal{J}_{n}}\left(1-\hat{\tau}_{\gamma}\right)^{-1} b_{\left(\gamma, \gamma^{\prime}\right)} \omega_{\gamma+4}\left(\left|x_{\gamma^{\prime}}(t)\right|\right) \\
& +\frac{1}{2} \sum_{\left(l, l^{\prime}\right) \in \mathcal{J}_{i} \times \mathcal{J}_{i}} d_{\left(l, l^{\prime}\right)}(t)\left\{\omega_{3}\left(\left\|x_{t}\left(-\tau_{l}(t)\right)\right\|\right) \omega_{3}\left(\left\|x_{t}\left(-\tau_{l^{\prime}}(t)\right)\right\|\right)\right\}^{\frac{1}{2}} \\
& +\sum_{k \in \mathcal{J}_{m}}\left[u_{k}(t)+p_{k}\left(t, x(t), x_{t}\left(-\tau_{1}(t)\right), \ldots, x_{t}\left(-\tau_{i}(t)\right), u(t)\right)\right]\left(D_{g_{k}} v_{1}\right)\left(t, x_{t}\right)+\left(D_{q} v_{1}\right)\left(t, x_{t}\right) .
\end{aligned}
$$

Using the inequality (4),

$$
\begin{aligned}
\dot{v}_{1}\left(t, x_{t}\right) & +\dot{v}_{2}\left(t, x_{t}\right)+\dot{v}_{3}\left(t, x_{t}\right) \leq-c \omega_{3}(\|x(t)\|) \\
& +\frac{1}{2} \sum_{\left(l, l^{\prime}\right) \in \mathcal{J}_{i} \times \mathcal{J}_{i}} d_{\left(l, l^{\prime}\right)}(t)\left\{\omega_{3}\left(\left\|x_{t}\left(-\tau_{l}(t)\right)\right\|\right) \omega_{3}\left(\left\|x_{t}\left(-\tau_{l^{\prime}}(t)\right)\right\|\right)\right\}^{\frac{1}{2}} \\
& +\sum_{k \in \mathcal{J}_{m}}\left[u_{k}(t)+p_{k}\left(t, x(t), x_{t}\left(-\tau_{1}(t)\right), \ldots, x_{t}\left(-\tau_{i}(t)\right), u(t)\right)\right]\left(D_{g_{k}} v_{1}\right)\left(t, x_{t}\right)+\left(D_{q} v_{1}\right)\left(t, x_{t}\right) .
\end{aligned}
$$

Hence, evaluating along solutions to (1a-b), it is easily shown that, for almost all $t \in\left[t_{0}, \infty\right)$,

$$
\begin{aligned}
\dot{v}_{1}\left(t, x_{t}\right) & +\dot{v}_{2}\left(t, x_{t}\right)+\dot{v}_{3}\left(t, x_{t}\right)+\dot{v}_{4}\left(t, x_{t}\right) \leq-\left\langle w(t), L\left(\lambda_{1}, \ldots, \lambda_{i}\right) w(t)\right\rangle \\
& +\sum_{k \in \mathcal{J}_{m}}\left[u_{k}(t)+p_{k}\left(t, x(t), x_{t}\left(-\tau_{1}(t)\right), \ldots, x_{t}\left(-\tau_{i}(t)\right), u(t)\right)\right]\left(D_{g_{k}} v_{1}\right)\left(t, x_{t}\right)+\left(D_{q} v_{1}\right)\left(t, x_{t}\right) .
\end{aligned}
$$

where, with $\bar{c}=c-\frac{1}{2} \sum_{\gamma \in \mathcal{J}_{i}}\left(1-\hat{\tau}_{\gamma}\right)^{-1} \bar{d}_{(\gamma, \gamma)}$,

$$
L\left(\lambda_{1}, \ldots, \lambda_{i}\right):=\left[\begin{array}{ccccccc}
\bar{c}-\sum_{\gamma \in \mathcal{J}_{i}}\left(1-\hat{\tau}_{\gamma}\right)^{-1} \lambda_{\gamma} & 0 & \ldots & \ldots & \ldots & \ldots & 0 \\
0 & \lambda_{1} & -\frac{1}{2} \bar{d}_{(1,2)} & \ldots & \ldots & \ldots & -\frac{1}{2} \bar{d}_{(1, i)} \\
\vdots & -\frac{1}{2} \bar{d}_{(1,2)} & \lambda_{2} & \ddots & & & \vdots \\
\vdots & \vdots & \ddots & \ddots & \ddots & & \vdots \\
\vdots & \vdots & & \ddots & \ddots & \ddots & \vdots \\
\vdots & \vdots & & & \ddots & \ddots & -\frac{1}{2} \bar{d}_{(i-1, i)} \\
0 & -\frac{1}{2} \bar{d}_{(1, i)} & \ldots & \ldots & \ldots & -\frac{1}{2} \bar{d}_{(i-1, i)} & \lambda_{i}
\end{array}\right],
$$

and $w(t):=\left[\left\{\omega_{3}(\|x(t)\|)\right\}^{\frac{1}{2}}\left\{\omega_{3}\left(\left\|x_{t}\left(-\tau_{1}(t)\right)\right\|\right)\right\}^{\frac{1}{2}} \ldots\left\{\omega_{3}\left(\left\|x_{t}\left(\tau_{i}(t)\right)\right\|\right)\right\}^{\frac{1}{2}}\right]^{\mathrm{T}}$. As a consequence of $(30)$ and in view of Lemmas 1 and 2, the functional $v$, defined in (29), satisfies, along solutions to (1a-b), the inequality:

$$
\begin{aligned}
\dot{v}\left(t, x_{t}\right) \leq & -\left\langle w(t), R_{1} w(t)\right\rangle+\left(M_{2}(t)+N_{2}(t)\right) w(t)+\varepsilon(t) \\
& +\sum_{\gamma \in \mathcal{J}_{i}}\left(1-\hat{\tau}_{\gamma}\right)^{-1} \varsigma_{\gamma}\left\{\omega_{3}(\|x(t)\|)\right\}^{\frac{1}{2}}-\sum_{\gamma \in \mathcal{J}_{i}} \varsigma_{\gamma}\left\{\omega_{3}\left(\left\|x_{t}\left(\tau_{\gamma}(t)\right)\right\|\right)\right\}^{\frac{1}{2}},
\end{aligned}
$$

where $R_{1}:=L\left(\lambda_{1}, \ldots, \lambda_{i}\right)-M_{1}-N_{1}, M_{1}, M_{2}(t)$ are specified in (6) and (7), respectively, and $N_{1}, N_{2}(t)$ and $\varepsilon(t)$ are defined in (8). Selecting,

$$
\forall \gamma \in \mathcal{J}_{i}, \quad \varsigma_{\gamma}=\alpha(t) a_{\gamma}+\sum_{k \in \mathcal{J}_{m}} \alpha_{k}(t) \xi_{\gamma, k} \geq 0
$$


the delay terms in $\left(M_{2}(t)+N_{2}(t)\right) w(t)$, on the righthand side of $(31)$, are eliminated and thence

$$
\dot{v}\left(t, x_{t}\right) \leq-\left\langle w(t), R_{1} w(t)\right\rangle+S(t) w(t)+\varepsilon(t),
$$

where $S(t):=\left[\begin{array}{lll}S_{1}(t) & 0 \ldots 0\end{array}\right]$ and $S_{1}(t)=\sum_{\gamma \in \mathcal{I}_{i}}\left(1-\hat{\tau}_{\gamma}\right)^{-1}\left(\alpha(t) a_{\gamma}+\sum_{k \in \mathcal{J}_{m}} \alpha_{k}(t) \xi_{\gamma, k}\right)$.

Assuming that there exist $\lambda_{1}, \ldots, \lambda_{i}, \mu_{1}, \ldots, \mu_{m}>0$ such that $R_{1}>0$, then, since $R_{1}$ is positive definite and symmetric,

$$
\left\|R_{1}^{-1}\right\|^{-1}\|w(t)\|^{2} \leq\left\langle w(t), R_{1} w(t)\right\rangle \leq\left\|R_{1}\right\|\|w(t)\|^{2} .
$$

Consequently, along solutions to (1a-b),

$$
\dot{v}\left(t, x_{t}\right) \leq-\left\|R_{1}^{-1}\right\|^{-1} \omega_{3}(\|x(t)\|)+S_{1}(t)\left\{\omega_{3}(\|x(t)\|)\right\}^{\frac{1}{2}}+\varepsilon(t) .
$$

Since, $\forall t \in\left[t_{0}, \infty\right)$ and $k \in \mathcal{J}_{m}, 0 \leq \alpha_{k}(t) \leq \bar{\alpha}_{k}, 0 \leq \alpha(t) \leq \hat{\alpha}$ and $0<\varepsilon_{k}(t) \leq \epsilon$, then

$$
\dot{v}\left(t, x_{t}\right) \leq-\left\|R_{1}^{-1}\right\|^{-1} \omega_{3}(\|x(t)\|)+\left\|R_{2}\right\|\left\{\omega_{3}(\|x(t)\|)\right\}^{\frac{1}{2}}+\bar{\epsilon},
$$

where $R_{2}=\left[\begin{array}{lll}R_{21} & 0 \ldots 0\end{array}\right], R_{21}=\sum_{\gamma \in \mathcal{I}_{i}}\left(1-\hat{\tau}_{\gamma}\right)^{-1}\left(\hat{\alpha} a_{\gamma}+\sum_{k \in \mathcal{J}_{m}} \bar{\alpha}_{k} \xi_{\gamma, k}\right)$ and $\bar{\epsilon}:=\epsilon \sum_{k \in \mathcal{J}_{m}} \bar{\alpha}_{k}$. Thus, all local solutions emanating outside any compact set containing

$$
\mathcal{V}_{1}\left(\lambda_{1}, \ldots, \lambda_{i}, \mu_{1}, \ldots, \mu_{m}\right)=\left\{x \in \mathbb{R}^{n}:\left\{\omega_{3}(\|x\|)\right\}^{\frac{1}{2}} \leq \Upsilon\left(\lambda_{1}, \ldots, \lambda_{i}, \mu_{1}, \ldots, \mu_{m}\right)\right\},
$$

where

$$
\Upsilon_{1}\left(\lambda_{1}, \ldots, \lambda_{i}, \mu_{1}, \ldots, \mu_{m}\right)=\frac{1}{2}\left\|R_{1}^{-1}\right\|\left(\left\|R_{2}\right\|+\sqrt{\left\|R_{2}\right\|^{2}+4 \bar{\epsilon}\left\|R_{1}^{-1}\right\|^{-1}}\right),
$$

are bounded and, hence, these solutions can be continued indefinitely. Finally, as a consequence of (34), there exist $\pi_{3} \in \Omega$ such that

$$
\dot{v}\left(t, x_{t}\right)+\pi_{3}\left(\delta\left(x(t), \mathcal{V}_{1}\right)\right) \leq 0,
$$

under the dynamics of (1a-b) and $\mathcal{V}_{1}$ is invariant. Thus, all conditions of Theorem 2 hold.

\section{Appendix C Proof of Theorem 5}

Condition (i) has been previously verified (see Appendix B). Now consider condition (ii) of Theorem 2. In applying the Geršgorin Theorem 1 to the matrix $R_{1}=\left[r_{\gamma, \gamma^{\prime}}\right]$, for all $\gamma, \gamma^{\prime} \in \mathcal{I}_{i}$, the conditions, $\forall \gamma \in \mathcal{I}_{i}, r_{\gamma \gamma}>\sum_{\gamma^{\prime} \in \mathcal{I}_{i} \backslash\{\gamma\}}\left|r_{\gamma \gamma^{\prime}}\right|$ ensure that $R_{1}$ is positive definite, which gives rise to

$$
\left\{\begin{array}{l}
\forall \gamma \in \mathcal{J}_{i}, \quad \lambda_{\gamma}>\frac{1}{2} \sum_{\substack{\gamma^{\prime} \in \mathcal{J}_{i} \\
\gamma \neq \gamma^{\prime}}} \bar{d}_{\left(\gamma, \gamma^{\prime}\right)}+\frac{1}{2} \sum_{\gamma^{\prime} \in \mathcal{I}_{i}}\left(a_{\gamma} \beta_{\gamma^{\prime}}+a_{\gamma^{\prime}} \beta_{\gamma}\right)+\sum_{\gamma^{\prime} \in \mathcal{I}_{i}} \Psi_{\gamma, \gamma^{\prime}}\left(\mu_{1}, \ldots, \mu_{m}\right), \\
\bar{c}-\sum_{\gamma \in \mathcal{J}_{i}}\left(1-\hat{\tau}_{\gamma}\right)^{-1} \lambda_{\gamma}>\frac{1}{2} \sum_{\gamma^{\prime} \in \mathcal{I}_{i}}\left(a_{0} \beta_{\gamma^{\prime}}+a_{\gamma^{\prime}} \beta_{0}\right)+\sum_{\gamma^{\prime} \in \mathcal{I}_{i}} \Psi_{0, \gamma^{\prime}}\left(\mu_{1}, \ldots, \mu_{m}\right) .
\end{array}\right.
$$

Suppose $\bar{c}>\Gamma+\Psi\left(\mu_{1}, \ldots, \mu_{m}\right)$, where $\Gamma$ and $\Psi$ are defined in (12) and (13), respectively, that is $\bar{c}$ satisfies

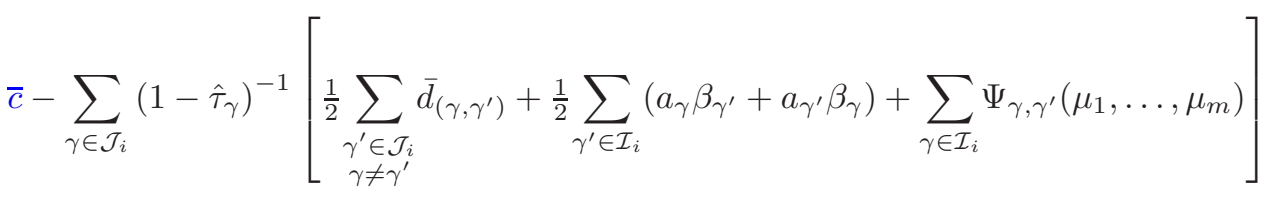

$$
\begin{aligned}
& >\frac{1}{2} \sum_{\gamma^{\prime} \in \mathcal{I}_{i}}\left(a_{0} \beta_{\gamma^{\prime}}+a_{\gamma^{\prime}} \beta_{0}\right)+\sum_{\gamma^{\prime} \in \mathcal{I}_{i}} \Psi_{0, \gamma^{\prime}}\left(\mu_{1}, \ldots, \mu_{m}\right),
\end{aligned}
$$

where $\Psi_{\gamma, \gamma^{\prime}}$ is defined in (9). Then it is possible to select, for all $\gamma \in \mathcal{J}_{i}$,

$$
\lambda_{\gamma}>\frac{1}{2} \sum_{\substack{\gamma^{\prime} \in \mathcal{J}_{i} \\ \gamma \neq \gamma^{\prime}}} \bar{d}_{\left(\gamma, \gamma^{\prime}\right)}+\frac{1}{2} \sum_{\gamma^{\prime} \in \mathcal{I}_{i}}\left(a_{\gamma} \beta_{\gamma^{\prime}}+a_{\gamma^{\prime}} \beta_{\gamma}\right)+\sum_{\gamma^{\prime} \in \mathcal{I}_{i}} \Psi_{\gamma, \gamma^{\prime}}\left(\mu_{1}, \ldots, \mu_{m}\right)
$$


such that

$$
\begin{aligned}
\bar{c} & -\sum_{\gamma \in \mathcal{J}_{i}}\left(1-\hat{\tau}_{\gamma}\right)^{-1}\left[\frac{1}{2} \sum_{\substack{\gamma^{\prime} \in \mathcal{J}_{i} \\
\gamma \neq \gamma^{\prime}}} \bar{d}_{\left(\gamma, \gamma^{\prime}\right)}+\frac{1}{2} \sum_{\gamma^{\prime} \in \mathcal{I}_{i}}\left(a_{\gamma} \beta_{\gamma^{\prime}}+a_{\gamma^{\prime}} \beta_{\gamma}\right)+\sum_{\gamma^{\prime} \in \mathcal{I}_{i}} \Psi_{\gamma, \gamma^{\prime}}\left(\mu_{1}, \ldots, \mu_{m}\right)\right] \\
> & \bar{c}-\sum_{\gamma \in \mathcal{J}_{i}}\left(1-\hat{\tau}_{\gamma}\right)^{-1} \lambda_{\gamma} \\
& >\frac{1}{2} \sum_{\gamma^{\prime} \in \mathcal{I}_{i}}\left(a_{0} \beta_{\gamma^{\prime}}+a_{\gamma^{\prime}} \beta_{0}\right)+\sum_{\gamma^{\prime} \in \mathcal{I}_{i}} \Psi_{0, \gamma^{\prime}}\left(\mu_{1}, \ldots, \mu_{m}\right)
\end{aligned}
$$

Therefore, it follows that there exists $\lambda_{1}, \ldots, \lambda_{i}, \mu_{1}, \ldots, \mu_{m}>0$ such that $R_{1}>0$.

Now $\Psi\left(\mu_{1}, \ldots, \mu_{m}\right)$ can be decomposed into $\hat{\Psi}\left(\mu_{1}, \ldots, \mu_{m}\right)+\tilde{\Psi}\left(\mu_{1}, \ldots, \mu_{m}\right)$, where $\hat{\Psi}\left(\mu_{1}, \ldots, \mu_{m}\right)$ does not have any local minimum and $\tilde{\Psi}\left(\mu_{1}, \ldots, \mu_{m}\right)$ has a local minimum with respect to $\mu_{k}$ provided there exists $\gamma \in \mathcal{I}_{i}$ such that $\xi_{\gamma, k} \neq 0$. Consequently, introducing the set $\mathcal{K}_{m}=\left\{k \in \mathcal{J}_{m}: \exists \gamma \in \mathcal{I}_{i}, \xi_{\gamma, k} \neq 0\right\}$, $\tilde{\Psi}\left(\mu_{1}, \ldots, \mu_{m}\right)$ and $\hat{\Psi}\left(\mu_{1}, \ldots, \mu_{m}\right)$ involve respective sums of the form $\sum_{k^{\prime} \in \mathcal{K}_{m}}(\cdot)$ and $\sum_{k^{\prime} \in \mathcal{J}_{m} \backslash \mathcal{K}_{m}}(\cdot)$. A calculation shows that $\tilde{\Psi}\left(\mu_{1}, \ldots, \mu_{m}\right)$ has a local minimum when $\mu_{k}=\mu_{k}^{*}, k \in \mathcal{K}_{m} \neq \emptyset$, where $\mu_{k}^{*}$ is defined in (14) and the minimum value of $\tilde{\Psi}\left(\mu_{1}, \ldots, \mu_{m}\right)$, given by $\tilde{\Psi}^{*}=\tilde{\Psi}\left(\mu_{1}^{*}, \ldots, \mu_{m}^{*}\right)$, is stated in (15).

Now consider the case when $\mu_{k} \in\left\{\mu_{k}: k \in \mathcal{J}_{m} \backslash \mathcal{K}_{m}\right\}$ or when $\mathcal{K}_{m}=\emptyset$. Let $\mu_{k}^{*}$ denote $\mu_{k}$ when $\mu_{k} \in\left\{\mu_{k}: k \in \mathcal{J}_{m} \backslash \mathcal{K}_{m}\right\}$ and it is designed, sufficiently large, so that

$$
\hat{\Psi}\left(\mu_{1}, \ldots, \mu_{m}\right)<\bar{c}-\Gamma-\tilde{\Psi}^{\dagger}
$$

where $\tilde{\Psi}^{\dagger}$ is defined in (16). Thus, it follows that if $\bar{c}>\Gamma+\hat{\Psi}\left(\mu_{1}, \ldots, \mu_{m}\right)+\tilde{\Psi}^{\dagger}$, then there exist $\lambda_{1}, \ldots, \lambda_{i}$, satisfying $(11)$, such that $R_{1}\left(\lambda_{1}, \ldots, \lambda_{i}, \mu_{1}, \ldots, \mu_{m}\right)>0$.

Considering the conditions on the parameters $\lambda_{\gamma}, \mu_{k}$, for $(\gamma, k) \in \mathcal{J}_{i} \times \mathcal{J}_{m}$, stated in (11), the following inequality is supposed to hold,

$$
\bar{c}>\Gamma+\tilde{\Psi}^{*},
$$

then $\mu_{k} \in\left\{\mu_{k}: k \in \mathcal{J}_{m} \backslash \mathcal{K}_{m}\right\}$ can be designed, sufficiently large, so that

$$
\hat{\Psi}<\bar{c}-\Gamma-\tilde{\Psi}^{*}
$$

i.e. $\bar{c}>\Gamma+\tilde{\Psi}^{*}+\hat{\Psi}$, and, hence, choosing $\lambda_{\gamma}, \gamma \in \mathcal{J}_{i}$, with respect to the inequalities (11), it follows that $R_{1}>0$.

The remaining part of the proof is analogous to that of Theorem 4.

\section{Appendix D Proof of Corollary 1}

It is only necessary to consider condition (ii) of Theorem 3, as condition (i) of Theorem 3 has already been verified in Appendix B.

It is assumed that there exist $\lambda_{1}, \ldots, \lambda_{i}, \mu_{1}, \ldots, \mu_{m}>0$ such that $R^{*}:=R_{1}>0$. From the proof of Theorem 4, in particular see (33), along solutions to (1a-b), the following holds:

$$
\dot{v}\left(t, x_{t}\right) \leq-\left\|R^{*}\right\|^{-1} \omega_{3}(\|x(t)\|)+S_{1}(t)\left\{\omega_{3}(\|x(t)\|)\right\}^{\frac{1}{2}}+\varepsilon(t),
$$

where $S_{1}$ is introduced in (32) and $\varepsilon$ is defined (8). Let $0<\Phi<1$, then (33) can be written as

$$
\dot{v}\left(t, x_{t}\right) \leq-(1-\Phi)\left\|R^{*}\right\|^{-1} \omega_{3}(\|x(t)\|)-\Phi\left\|R^{*}\right\|^{-1} \omega_{3}(\|x(t)\|)+S_{1}(t)\left\{\omega_{3}(\|x(t)\|)\right\}^{\frac{1}{2}}+\varepsilon(t) .
$$

Using the inequality $-a u^{2}+u v \leq v^{2} /(4 a)$, for $a \in \mathbb{R} \backslash\{0\}$ and $(u, v) \in \mathbb{R}^{2}$,

$$
-\Phi\left\|R^{*}\right\|^{-1} \omega_{3}(\|x(t)\|)+S_{1}(t)\left\{\omega_{3}(\|x(t)\|)\right\}^{\frac{1}{2}} \leq \frac{S_{1}^{2}(t)}{4 \Phi\left\|R^{*}\right\|^{-1}} .
$$


Hence, (35) becomes

$$
\dot{v}\left(t, x_{t}\right) \leq-(1-\Phi)\left\|R^{*}\right\|^{-1} \omega_{3}(\|x(t)\|)+\frac{S_{1}^{2}(t)}{4 \Phi\left\|R^{*}\right\|^{-1}}+\varepsilon(t) .
$$

Note that, since $S_{1}(t)=\sum_{\gamma \in \mathcal{I}_{i}}\left(1-\hat{\tau}_{\gamma}\right)^{-1}\left(\alpha(t) a_{\gamma}+\sum_{k \in \mathcal{J}_{m}} \alpha_{k}(t) \xi_{\gamma, k}\right)$,

$$
\frac{S_{1}^{2}(t)}{4 \Phi\left\|R^{*}\right\|^{-1}}+\varepsilon(t) \leq \chi:=\sum_{\gamma \in \mathcal{I}_{i}}\left(1-\hat{\tau}_{\gamma}\right)^{-1}\left(\hat{\alpha} a_{\gamma}+\sum_{k \in \mathcal{J}_{m}} \bar{\alpha}_{k} \xi_{\gamma, k}\right) /\left(4 \Phi\left\|R^{*}\right\|^{-1}\right)+\epsilon \sum_{k \in \mathcal{J}_{m}} \bar{\alpha}_{k} .
$$

Since $\omega_{3} \in \Omega$, then $\eta>0$ can be determined, sufficiently large, such that $(1-\Phi)\left\|R^{*}\right\|^{-1} \omega_{3}(\eta)>\chi$. Define $t \mapsto \varphi(t):=\left(S_{1}^{2}(t) /\left(4 \Phi\left\|R^{*}\right\|^{-1}\right)+\varepsilon(t)\right) /\left[(1-\Phi)\left\|R^{*}\right\|^{-1} \omega_{3}(\eta)\right]$, then

$$
|\varphi(t)| \leq \frac{\chi}{(1-\Phi)\left\|R^{*}\right\|^{-1} \omega_{3}(\eta)}<1
$$

and (36) can be expressed in the form

$$
\dot{v}\left(t, x_{t}\right)+(1-\Phi)\left\|R^{*}\right\|^{-1} \omega_{3}(\|x(t)\|) \leq(1-\Phi)\left\|R^{*}\right\|^{-1} \omega_{3}(\eta) \varphi(t) .
$$

For all $k \in \mathcal{J}_{m}$, since $\varepsilon_{k} \in L^{2}\left(t_{0}, \infty\right)$ and $\alpha_{k} \in L^{2}\left(t_{0}, \infty\right)$, then, in view of $(8), \varepsilon \in L^{1}\left(t_{0}, \infty\right)($ see $\S 6.9$, Chapter 6 in [1]). Similarly, since $\alpha \in L^{2}\left(t_{0}, \infty\right)$ it follows that $S_{1} \in L^{2}\left(t_{0}, \infty\right)$ and so $S_{1}^{2} \in L^{1}\left(t_{0}, \infty\right)$. Hence, $\varphi \in L^{1}\left(t_{0}, \infty\right)$ and, therefore, all the conditions of Theorem 3 hold, with $\pi_{3} \equiv(1-\Phi)\left\|R^{*}\right\|^{-1} \omega_{3}$.

\section{Appendix E Proof of Lemma 3}

Using H3a), H4a) and in view of (18) ( see (27) with $\mu_{k}=0 \forall k$ ), for all $\left(t, x(t), x_{t}, u(t)\right) \in\left[t_{0}, \infty\right) \times$ $\mathbb{R}^{n} \times \mathcal{Q}_{A, \bar{\tau}}^{n} \times \mathbb{R}^{m}$,

$$
\begin{aligned}
{\left[u_{k}(t)+\right.} & \left.p_{k}\left(t, x(t), x_{t}\left(-\tau_{1}(t)\right), \ldots, x_{t}\left(-\tau_{i}(t)\right), u(t)\right)\right]\left(D_{g_{k}} v_{1}\right)\left(t, x_{t}\right) \\
\leq & \left|\left(D_{g_{k}} v_{1}\right)\left(t, x_{t}\right)\right| \sum_{\gamma \in \mathcal{I}_{i}} \beta_{\gamma, k}\left\{\omega_{3}\left(\left\|x_{t}\left(-\tau_{\gamma}(t)\right)\right\|\right)\right\}^{\frac{1}{2}} \\
& +\alpha_{k}(t) \varepsilon_{k}(t)+\alpha_{k}(t) \sum_{\gamma \in \mathcal{I}_{i}} \xi_{\gamma, k}\left\{\omega_{3}\left(\left\|x_{t}\left(-\tau_{\gamma}(t)\right)\right\|\right)\right\}^{\frac{1}{2}} .
\end{aligned}
$$

Hence,

$$
\begin{aligned}
A w(t) \rho_{k}\left|\left(D_{g_{k}} v_{1}\right)\left(t, x_{t}\right)\right|+ & {\left[u_{k}(t)+p_{k}\left(t, x(t), x_{t}\left(-\tau_{1}(t)\right), \ldots, x_{t}\left(-\tau_{i}(t)\right), u(t)\right)\right]\left(D_{g_{k}} v_{1}\right)\left(t, x_{t}\right) } \\
\leq & \left|\left(D_{g_{k}} v_{1}\right)\left(t, x_{t}\right)\right| \sum_{\gamma \in \mathcal{I}_{i}}\left(a_{\gamma} \rho_{k}+\beta_{\gamma, k}\right)\left\{\omega_{3}\left(\left\|x_{t}\left(-\tau_{\gamma}(t)\right)\right\|\right)\right\}^{\frac{1}{2}} \\
& +\alpha_{k}(t) \varepsilon_{k}(t)+\alpha_{k}(t) \sum_{\gamma \in \mathcal{I}_{i}} \xi_{\gamma, k}\left\{\omega_{3}\left(\left\|x_{t}\left(-\tau_{\gamma}(t)\right)\right\|\right)\right\}^{\frac{1}{2}} .
\end{aligned}
$$

In view of $\mathrm{H} 6$, it follows that, for all $k \in \mathcal{J}_{m}$,

$$
\begin{aligned}
& A w(t) \rho_{k}\left|\left(D_{g_{k}} v_{1}\right)\left(t, x_{t}\right)\right|+\left[u_{k}(t)+p_{k}\left(t, x(t), x_{t}\left(-\tau_{1}(t)\right), \ldots, x_{t}\left(-\tau_{i}(t)\right), u(t)\right)\right]\left(D_{g_{k}} v_{1}\right)\left(t, x_{t}\right) \\
& \leq \sum_{\gamma \in \mathcal{I}_{i}}\left(a_{\gamma} \rho_{k}+\beta_{\gamma, k}\right)\left\{\omega_{3}\left(\left\|x_{t}\left(-\tau_{\gamma}(t)\right)\right\|\right)\right\}^{\frac{1}{2}} \sum_{\gamma^{\prime} \in \mathcal{I}_{i}} \chi_{\gamma^{\prime}, k}\left\{\omega_{3}\left(\left\|x_{t}\left(-\tau_{\gamma^{\prime}}(t)\right)\right\|\right)\right\}^{\frac{1}{2}} \\
& \quad+\alpha_{k}(t) \varepsilon_{k}(t)+\alpha_{k}(t) \sum_{\gamma \in \mathcal{I}_{i}} \xi_{\gamma, k}\left\{\omega_{3}\left(\left\|x_{t}\left(-\tau_{\gamma}(t)\right)\right\|\right)\right\}^{\frac{1}{2}} .
\end{aligned}
$$

The result then follows. 


\section{Appendix F Proof of Lemma 4}

Geršgorin's Theorem 1 applied to the matrix $\bar{R}_{1}=\left[\bar{r}_{\gamma, \gamma^{\prime}}\right]$, for all $\gamma, \gamma^{\prime} \in \mathcal{I}_{i}$, shows that $\bar{R}_{1}>0$ if, $\forall \gamma \in \mathcal{I}_{i}, \bar{r}_{\gamma \gamma}>\sum_{\gamma^{\prime} \in \mathcal{I}_{i} \backslash\{\gamma\}}\left|\bar{r}_{\gamma \gamma^{\prime}}\right|$, namely

$$
\left\{\begin{array}{l}
\forall \gamma \in \mathcal{J}_{i}, \quad \lambda_{\gamma}>\frac{1}{2} \sum_{\substack{\gamma^{\prime} \in \mathcal{J}_{i} \\
\gamma \neq \gamma^{\prime}}} \bar{d}_{\left(\gamma, \gamma^{\prime}\right)}+\frac{1}{2} \sum_{\gamma^{\prime} \in \mathcal{I}_{i}}\left(a_{\gamma} \beta_{\gamma^{\prime}}+a_{\gamma^{\prime}} \beta_{\gamma}\right)+\sum_{\gamma^{\prime} \in \mathcal{I}_{i}} \Lambda_{\gamma, \gamma^{\prime}}, \\
\text { and } \bar{c}-\sum_{\gamma \in \mathcal{J}_{i}}\left(1-\hat{\tau}_{\gamma}\right)^{-1} \lambda_{\gamma}>\frac{1}{2} \sum_{\gamma^{\prime} \in \mathcal{I}_{i}}\left(a_{0} \beta_{\gamma^{\prime}}+a_{\gamma^{\prime}} \beta_{0}\right)+\sum_{\gamma^{\prime} \in \mathcal{I}_{i}} \Lambda_{0, \gamma^{\prime}} .
\end{array}\right.
$$

Suppose $\bar{c}>\Gamma+\Lambda$, where $\Gamma$ and $\Lambda$ are defined in (12) and (21), respectively, that is $c$ satisfies

$$
\begin{aligned}
& \bar{c}-\sum_{\gamma \in \mathcal{J}_{i}}\left(1-\hat{\tau}_{\gamma}\right)^{-1}\left[\frac{1}{2} \sum_{\substack{\gamma^{\prime} \in \mathcal{J}_{i} \\
\gamma \neq \gamma^{\prime}}} \bar{d}_{\left(\gamma, \gamma^{\prime}\right)}+\frac{1}{2} \sum_{\gamma^{\prime} \in \mathcal{I}_{i}}\left(a_{\gamma} \beta_{\gamma^{\prime}}+a_{\gamma^{\prime}} \beta_{\gamma}\right)+\sum_{\gamma \in \mathcal{I}_{i}} \Lambda_{\gamma, \gamma^{\prime}}\right] \\
& >\frac{1}{2} \sum_{\gamma^{\prime} \in \mathcal{I}_{i}}\left(a_{0} \beta_{\gamma^{\prime}}+a_{\gamma^{\prime}} \beta_{0}\right)+\sum_{\gamma^{\prime} \in \mathcal{I}_{i}} \Lambda_{0, \gamma^{\prime}},
\end{aligned}
$$

where $\Lambda_{\gamma, \gamma^{\prime}}$ is defined in (19). Then it is possible to select, for all $\gamma \in \mathcal{J}_{i}$,

$$
\lambda_{\gamma}>\frac{1}{2} \sum_{\substack{\gamma^{\prime} \in \mathcal{J}_{i} \\ \gamma \neq \gamma^{\prime}}} \bar{d}_{\left(\gamma, \gamma^{\prime}\right)}+\frac{1}{2} \sum_{\gamma^{\prime} \in \mathcal{I}_{i}}\left(a_{\gamma} \beta_{\gamma^{\prime}}+a_{\gamma^{\prime}} \beta_{\gamma}\right)+\sum_{\gamma^{\prime} \in \mathcal{I}_{i}} \Lambda_{\gamma, \gamma^{\prime}}
$$

such that

$$
\begin{aligned}
\bar{c} & -\sum_{\gamma \in \mathcal{J}_{i}}\left(1-\hat{\tau}_{\gamma}\right)^{-1}\left[\frac{1}{2} \sum_{\substack{\gamma^{\prime} \in \mathcal{J}_{i} \\
\gamma \neq \gamma^{\prime}}} \bar{d}_{\left(\gamma, \gamma^{\prime}\right)}+\frac{1}{2} \sum_{\gamma^{\prime} \in \mathcal{I}_{i}}\left(a_{\gamma} \beta_{\gamma^{\prime}}+a_{\gamma^{\prime}} \beta_{\gamma}\right)+\sum_{\gamma^{\prime} \in \mathcal{I}_{i}} \Lambda_{\gamma, \gamma^{\prime}}\right] \\
> & \bar{c}-\sum_{\gamma \in \mathcal{J}_{i}}\left(1-\hat{\tau}_{\gamma}\right)^{-1} \lambda_{\gamma} \\
& >\frac{1}{2} \sum_{\gamma^{\prime} \in \mathcal{I}_{i}}\left(a_{0} \beta_{\gamma^{\prime}}+a_{\gamma^{\prime}} \beta_{0}\right)+\sum_{\gamma^{\prime} \in \mathcal{I}_{i}} \Lambda_{0, \gamma^{\prime}}
\end{aligned}
$$

For this choice of $\lambda_{\gamma}$, conditions (37) hold and so $\bar{R}_{1}>0$.

\section{References}

[1] Bartle, R.G., 1966, The elements of integration (New York, U.S.A.: John Wiley \& Sons).

[2] Chen, W.H., Guan, Z.H., and Lu, L., 2004, Delay-dependent output feedback guaranteed cost control for uncertain time-delay systems. Automatica, 40, 1263-1268.

[3] Clarkson, I.D., and Goodall, D.P., 2000, Stabilizing output feedback controls for a class of perturbed nonlinear dynamical delay systems. Proceedings of the 14th International Symposium on Mathematical Theory of Networks and Systems, MTNS 2000 (CD-Rom), Perpignan, France.

[4] Clarkson, I.D., and Goodall, D.P., 2001, Robust stabilizing feedback for a class of imperfectly known nonlinear systems with multiple time-delays. IMA Journal of Mathematical Control and Information, 18, No. 3, 355-379.

[5] de Oliveira, M.C., and Geromel, J.C., 2004, Synthesis of non-rational controllers for linear delay systems. Automatica, 40, No. 2, 171-188. 
[6] Fridman, E., Shaked, U., 2003, Delay-dependent stability and $H_{\infty}$ control: constant and timevarying delays. International Journal of Control, 76, No. 1, 48-60.

[7] Germani, A., Manes, C., and Pepe, P., 2002, A new approach to state observation of nonlinear systems with delayed output. IEEE Transactions on Automatic Control, 47, No. 1, 96-101.

[8] Goodall, D.P., 2007, Stabilizing inputs for implicit functional differential equations of the neutral type with unknown time-varying delays. Journal of Dynamical and Control Systems, 13, No. 2, $177-215$.

[9] Goodall, D.P., 2006, Robust stabilizers for an implicit dynamical delay system of the neutral type. International Journal of Robust and Nonlinear Control, Vol. 16, No. 4, 215-241.

[10] Gu, K., and Niculescu, S.I., 2003, Survey on recent results in the stability and control of time-delay systems. Journal of Dynamic Systems, Measurement, and Control, 125, 158-165.

[11] Hale, J.K., and Verduyn Lunel, S.M., 1993, Introduction to functional differential equations (New York, U.S.A.: Springer-Verlag).

[12] Haurani, H., Michalska, H.H., and Boulet, B., 2004, Robust output feedback stabilization of uncertain time-varying state-delayed systems with saturating actuators. International Journal of Control, 77, No. 4, 399-414.

[13] He, Y., Wang, Q.-G., Lin, C., and Wu, M., 2007, Delay-range-dependent stability for systems with time-varying delay. Automatica, 43, 371-376.

[14] Hennet, J.C., and Tarbouriech, S., 1997, Stability and stabilization of delay differential systems. Automatica, 33, 347-354.

[15] Kao, C.-Y., and Rantzer, A., 2007, Stability analysis of systems with uncertain time-varying delays. Automatica, 43, 959-970.

[16] Lam, J., Gao, H., and Wang, C., 2007, Stability analysis for continuous systems with two additive time-varying delay components. Systems and Control Letters, 56, 16-24.

[17] Michel, A.N., Wang, K., and Hu, B., 2001, Qualitative theory of dynamical systems. (New York, U.S.A.: Marcel Dekker).

[18] Mirkin, B.M., and Gutman, P.E., 2003, Output-feedback model reference adaptative control for continuous state delay systems. Journal of Dynamical Systems, Measurement, and Control, 125, $257-261$.

[19] Park, J.H., 2004, Design of a dynamic output feedback controller for a class of neutral systems with discrete and distributed delays IEE Proceedings on Control Theory and Applications, 151, No. 5, $610-614$.

[20] Qu, Z, 1998, Robust control of nonlinear uncertain systems (New York, U.S.A.: John Wiley \& Sons).

[21] Richard, J.P., 2003, Time-delay systems: an overview of some recent advances and open problems. Automatica, 39, 1667-1694.

[22] Su, H.L., Hu, J., and Chu, J., 2001, Output feedback stabilization of uncertain time-delay systems containing saturating actuators. Developments in Chemical Engineering and Mineral Processing, 9, $183-190$.

[23] Tarbouriech, S., and Garcia, G., (Editors), 1997, Control of uncertain systems with bounded inputs (Lecture Notes in Control and Information Sciences, Vol. 227). (Berlin/Heidelberg, Germany: Springer-Verlag).

[24] Varga, R.S, 2004, Gersgorin and his circles (Berlin/Heidelberg, Germany: Springer).

[25] Wu, H., and Mizukami, K., 1993, Exponential stability of a class of nonlinear dynamical systems with uncertainties. Systems and Control Letters, 21, 307-313. 
[26] Wu, W., and Chou, Y.S., 1996, Output tracking control of uncertain nonlinear systems with an input delay. IEE Proc.-Control Theory and Applications, 143, No. 4, 309-318.

[27] Zhang, X., and Cheng, Z., 2005, Output feedback stabilization of nonlinear systems with delays in the input. Journal of Applied Mathematics and Computation, 167, 1405-1412.

[28] Zhang, X., Gao, H., and Zhang, C., 2006, Global asymptotic stabilization of feedforward nonlinear systems with a delay in the input. International Journal of Systems Science, 37, No. 3, 141-148. 